\title{
Travel time and trajectory moments of conservative solutes in two-dimensional convergent flows
}

\author{
M. Riva ${ }^{\text {a }}$, X. Sánchez-Vila ${ }^{\text {b** }}$, A. Guadagnini ${ }^{a}$, \\ M. De Simoni ${ }^{\mathrm{a}}$, M. Willmann ${ }^{\mathrm{b}}$ \\ a Dipartimento Ingegneria Idraulica, Ambientale, Infrastrutture Viarie, Rilevamento, Politecnico di Milano, \\ Piazza L. Da Vinci 32, 20133 Milano, Italy \\ ${ }^{\mathrm{b}}$ Department of Geotechnical Engineering and Geosciences, Technical University of Catalonia, \\ Gran Capità S/N, 08034 Barcelona, Spain
}

Received 30 April 2004; received in revised form 19 April 2005; accepted 1 June 2005

Available online 10 October 2005

\begin{abstract}
We address advective transport of a solute traveling toward a single pumping well in a twodimensional randomly heterogeneous aquifer. The two random variables of interest are the trajectory followed by an individual particle from the injection point to the well location and the particle travel time under steady-state conditions. Our main objective is to derive the predictors of trajectory and travel time and the associated uncertainty, in terms of their first two statistical moments (mean and variance). We consider a solute that undergoes mass transfer between a mobile and an immobile zone. Based on Lawrence et al. [Lawrence, A.E., Sánchez-Vila, X., Rubin, Y., 2002. Conditional moments of the breakthrough curves of kinetically sorbing solute in heterogeneous porous media using multirate mass transfer models for sorption and desorption. Water Resour. Res. 38 (11), 1248, doi:10.1029/ 2001WR001006.], travel time moments can be written in terms of those of a conservative solute times a deterministic quantity. Moreover, the moments of solute particles trajectory do not depend on mass transfer processes. The resulting mean and variance of travel time and trajectory for a conservative species can be written as functions of the first, second moments and cross-moments of trajectory and velocity components. The equations are developed from a consistent second order expansion in $\sigma_{Y}$ (standard deviation of the natural logarithm of hydraulic conductivity). Our solution can be completely integrated with the moment equations of groundwater flow of Guadagnini and Neuman [Guadagnini, A., Neuman, S.P., 1999a. Nonlocal and localized analyses of conditional mean steady state flow in bounded,
\end{abstract}

\footnotetext{
* Corresponding author.

E-mail address: xavier.sanchez-vila@upc.es (X. Sánchez-Vila).
} 
randomly non uniform domains 1. Theory and computational approach. Water Resour. Res. 35(10), 2999-3018.,Guadagnini, A., Neuman, S.P., 1999b. Nonlocal and localized analyses of conditional mean steady state flow in bounded, randomly non uniform domains 2. Computational examples. Water Resour. Res. 35(10), 3019-3039.], it is free of distributional assumptions regarding the log conductivity field, and formally includes conditioning. We present analytical expressions for the unconditional case by making use of the results of Riva et al. [Riva, M., Guadagnini, A., Neuman, S.P., Franzetti, S., 2001. Radial flow in a bounded randomly heterogeneous aquifer. Transport in Porous Media 45, 139-193.]. The quality of the solution is supported by numerical Monte Carlo simulations. Potential uses of this work include the determination of aquifer reclamation time by means of a single pumping well, and the demarcation of the region potentially affected by the presence of a contaminant in the proximity of a well, whenever the aquifer is very thin and Dupuit-Forchheimer assumption holds.

(C) 2005 Elsevier B.V. All rights reserved.

Keywords: Groundwater flow and transport; Porous media; Travel time; Statistical moments; Model uncertainty

\section{Introduction}

A plausible scenario in risk assessment for repositories consists of drilling a well in the vicinity of the repository many years after its closure, when the buried material is still hazardous. If after several years of pumping the engineering barrier fails and some contaminant is released,

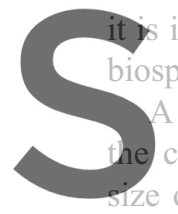
important to evaluate osphere. completely different of the plume, the tym
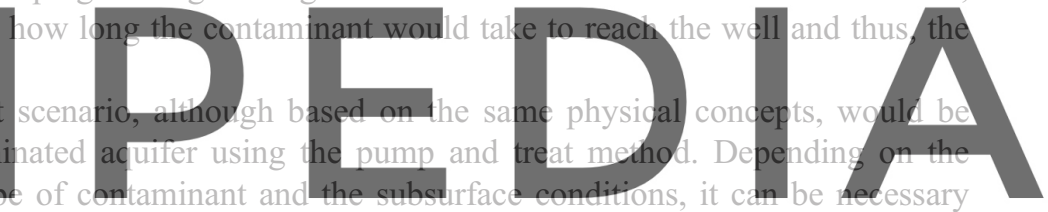

to pump until some remediation goals are met. The relevant question in this scenario would

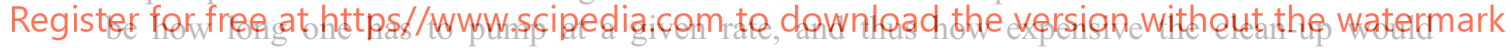
be.

Both mentioned scenarios can arise in several geological settings, including both porous and fractured formations. It is recognized that prediction of transport of solutes in a real flow configuration is never certain. The uncertainty associated to the parameters governing groundwater flow and solute transport is conveniently tackled within a stochastic framework. The latter recognizes that in natural aquifers it is not possible to describe deterministically either hydrogeological parameters or the space-time distribution of the state variables of interest. Instead, the stochastic approach offers the appealing alternative of characterizing these quantities in probabilistic terms, by means of either their complete probability distribution or a few, significant statistical moments, particularly the low order ones.

Here, we focus on the impact of the porous medium heterogeneity on contaminant transport under convergent flow conditions, such as those created by a single pumping well. It is well known that in the presence of a single pumping well creating a purely radial flow within a homogeneous aquifer (a) the trajectory of any particle injected in the domain, in the absence of dispersion, is a straight line connecting the point of injection and the well, (b) the velocity of the particle at any given point is inversely proportional to the distance from the well and directly proportional to the pumping rate, and (c) the travel time of a conservative solute is proportional to the square of the distance from the well and inversely proportional to the pumping rate. These simple results are not amenable to direct translation to a heterogeneous 
formation, where local velocities, trajectories and solute travel time become uncertain. This work is aimed to deriving unbiased predictors of the two latter quantities (rendered by their mean values), together with the associated predictive uncertainty (as rendered by second order moments, i.e., variances).

The problem of determining statistical moments of travel time has been typically analyzed for the particular case of mean uniform flow conditions in infinite domains (e.g. Shapiro and Cvetkovic, 1988; Dagan et al., 1992; Cvetkovic et al., 1996; Rajaram, 1997; Guadagnini et al., 2003). In these works either conservative or sorptive solutes are considered. Only a few results are available for more complex flow conditions. Dagan and Indelman (1999) determine mean and variance of travel time for a steady-state flow between a fully penetrating recharging and pumping well (doublet) that takes place in an infinite aquifer upon assuming that the trajectory of a tracer particle coincides with that observed in a homogeneous field. Zhang et al. (2000) provide the expressions for mean and variance of travel time and trajectory of an ideal solute particle in a two-dimensional non-uniform mean flow in the absence of source terms. Lessoff and Indelman (2004) develop an expression for the variance of travel time of a conservative solute to a fully penetrating well in a three-dimensional infinite domain. Their expression is based on an approximation of first order (in terms of the variance of the natural logarithm of hydraulic conductivity) that is valid for small anisotropy ratio (vertical to horizontal) of the loghydraulic conductivity correlation function. Their approximation does not hold for twodimensional media.

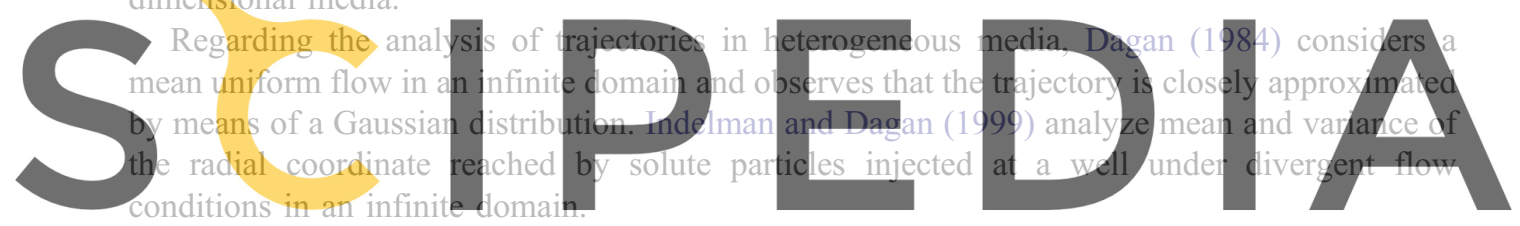

Most of the existing work on transport under convergent flow conditions has focused on well

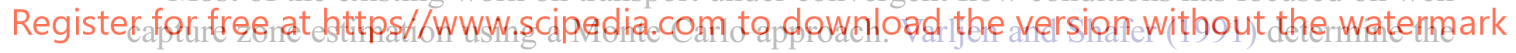

1 - and 10-year probabilistic capture regions in a rectangular domain, in the presence of random, conditional fields of hydraulic conductivity. Franzetti and Guadagnini (1996) and Guadagnini and Franzetti (1999) study the influence of unconditional heterogeneity of transmissivity on time-related capture zones and well catchment delineation for a single well operating under uniform background flow. Riva et al. (1999) consider a similar problem for purely convergent flow conditions. Van Leeuwen et al. (2000) and Feyen et al. (2001), respectively evaluate the effect of conditioning upon transmissivity or head measurements on delineation of well capture zones.

Travel time moments of sorptive solutes under non-uniform flow conditions have been addressed by Cvetkovic et al. (1998) in an unconditional frame. Lawrence et al. (2002) and Sánchez-Vila and Rubin (2003) tackled the problem in a conditional framework: the former consider sorption parameters as deterministic, while the latter model them as random space functions. Their main finding is the conclusion that the statistical moments of travel time of a non-conservative solute can be expressed in terms of those of a conservative one. Lawrence et al. (2002) and Sánchez-Vila and Rubin (2003) also stated that once the general relationship between moments of conservative and non-conservative solutes has been established the next step to be carried out would be the evaluation of the actual travel time moments of conservative solutes for different flow configurations. In particular, Sánchez-Vila and Rubin (2003) studied in detail the statistical moments of travel time for reactive solutes in a convergent flow situation (like the one we tackle in this paper) and evaluated the travel time 
moments for conservative solutes on the basis of numerical Monte Carlo simulations available in the literature.

Guadagnini et al. (2003) developed a fully non-linear mathematical formalism to calculate mean and variance of travel time and trajectory of a conservative solute in a twodimensional domain under general non-uniform flow conditions. Sánchez-Vila and Guadagnini (2005) extended the formal expressions of Guadagnini et al. (2003) to threedimensional random flows and solved analytically the equations satisfied by mean and (cross-co)variance of particles travel times and trajectories under the special case of uniform flow in the mean.

Here we start from the work of Guadagnini et al. (2003) and present analytical expressions for the mean and variance of travel time of particles traveling under steady-state two-dimensional convergent flow conditions. We consider Dirichlet-type boundary conditions and assume that the head drawdown is negligible at a given distance. The choice of these conditions is based on the work of Sánchez-Vila et a1. (1999), who noted that for large pumping times the differential drawdown between the pumping point and any observation point becomes constant with time. Thus, since the shape of the drawdown surface does not vary with time, one can define a curve with all points having the same drawdown. From a mathematical standpoint, this is analogous to assigning zero drawdown to this curve; its shape would depend on the actual spatial distribution of transmissivity, but in the mean it will be a circumference. Furthermore, we note that this condition is equivalent to the one considered by Thiem (1906) from which it is possible to derive the travel time of particl results,
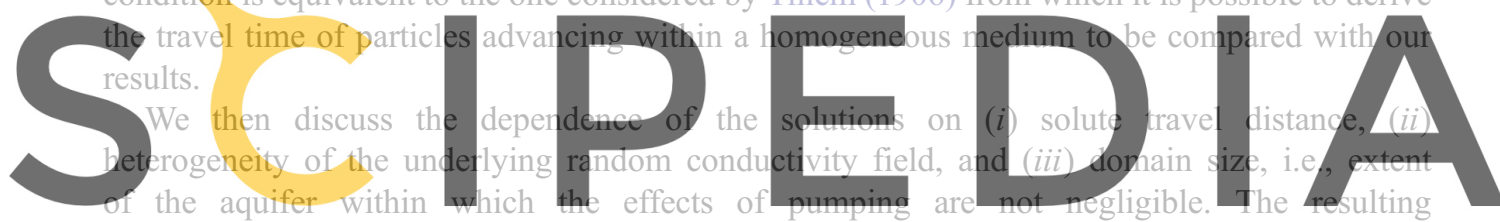

expressions are then compared with an extensive suite of Monte Carlo simulations. The

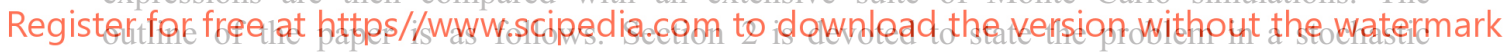

framework. It is followed by two sections devoted to the evaluation of the ensemble moments of particle trajectory (Section 3) and travel time (Section 4) for conservative solutes. Finally, Section 5 presents the comparison between the analytical results and numerical Monte Carlo simulations.

\section{Statement of the problem}

We consider incompressible groundwater steady state convergent flow created by a well of zero radius, located at the center of a circular randomly heterogeneous porous domain of radial extent $L$. The well pumps at a constant deterministic rate, $Q$. Hydraulic head remains at a constant deterministic value along the outer circular boundary. Riva et al. (2001) derived analytically the statistical moments of hydraulic head and specific flux for this type of flow configuration. Here the variables of interest are travel time and trajectory of non-conservative solute particles released at time $t_{0}=0$ at a general point of polar coordinates $\mathbf{r}_{0} \equiv\left(r_{0}, \theta_{0}\right)$, where radial distances are measured starting from the well location.

The solute is considered to undergo mass transfer processes between a mobile and an immobile phases. Assuming the parameters governing mass transfer are homogeneous, Lawrence et al. (2002) found that the first two statistical moments of travel time for nonconservative solutes can be expressed in terms of those of conservative solutes (Lawrence et al., 2002; their Eqs. (14) and (15)). However, formal derivation and evaluation of the latter were not 
provided. As the trajectory moments are also independent of mass transfer processes, in the remaining of the paper we will only analyze transport of conservative species.

The starting point, then, is Darcy's law, which relates the fluid velocity $\mathbf{V}(\mathbf{r})$ at a vector location $\mathbf{r} \equiv(r, \theta)$ to the local hydraulic head, $h(\mathbf{r})$, through the local hydraulic conductivity $K(\mathbf{r})$ :

$$
\mathbf{V}(\mathbf{r})=\frac{\mathbf{q}(\mathbf{r})}{n(\mathbf{r})}=-\frac{K(\mathbf{r})}{n(\mathbf{r})} \nabla h(\mathbf{r})
$$

where $\mathbf{q}(\mathbf{r})$ is the specific flux, $n$ is the effective porosity. Porosity is taken as a constant, due to its relatively modest spatial variability (see e.g. Varljen and Shafer, 1991). The trajectory of a conservative solute traveling towards the well is rendered by the kinematic equations:

$$
\mathrm{d} \mathbf{r}=(\mathrm{d} r, r \mathrm{~d} \theta)=\left\{V_{r}(r, \theta) \mathrm{d} t, V_{\theta}(r, \theta) \mathrm{d} t\right\}
$$

where $r$ is the radial distance from the well, $V_{r}$ and $V_{\theta}$ are respectively the components of the velocity vector, $\mathbf{V}(\mathbf{r})$, along the radial and tangential directions. The solution of Eq. (2) provides the position reached at time $t$ by the particle injected at location $\mathbf{r}=\mathbf{r}_{0}$ at $t=t_{0}$ and is given in parametric form by

\section{$r=r\left(t, t_{0}\right) ; \quad \theta=\theta\left(t, t_{0}\right)$}
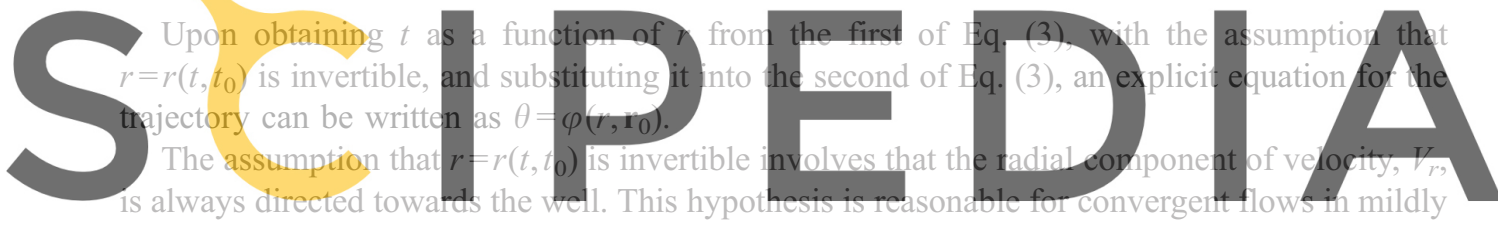

heterogeneous conductivity domains. The differential equation for the projection of the

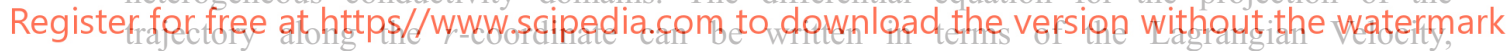

$V_{r}\left(r, \varphi\left(r, \mathbb{r}_{0}\right)\right)$, as:

$$
\mathrm{d} t=\frac{\mathrm{d} r}{V_{r}\left(r, \varphi\left(r, \mathbf{r}_{0}\right)\right)} .
$$

Under purely convergent flow conditions the well location is the endpoint of all particle trajectories. The travel time to the well, that is the time required for a particle starting at location $\mathbf{r}=\mathbf{r}_{0}$ at $t=t_{0}=0$ to reach the well, can be expressed upon integration of Eq. (4). Since we treat $K$ as a Random Spatial Function, the Eulerian velocity, $\mathbf{V}(r, \theta)$, the Lagrangian velocity, $\mathbf{V}\left(r, \varphi\left(r, \mathbf{r}_{0}\right)\right)$, the solute travel time, $t\left(r=0, \mathbf{r}_{0}\right)$, and the particle trajectory $\varphi\left(r, \mathbf{r}_{0}\right)$ also become random functions.

Here we model the natural logarithm of $K, Y(\mathbf{r})=\ln K(\mathbf{r})$, as a statistically homogeneous and isotropic Random Spatial Function, with covariance between two points $\mathbf{r}_{\mathrm{I}} \equiv\left(r_{\mathrm{I}}, \theta_{\mathrm{I}}\right)$ and $\mathbf{r}_{\mathrm{II}} \equiv\left(r_{\mathrm{II}}, \theta_{\mathrm{II}}\right)$ given by

$$
C_{Y}\left(\xi_{\mathrm{I}}, \xi_{\mathrm{II}}, \theta_{\mathrm{I}}, \theta_{\mathrm{II}}\right)=C_{Y}\left(\xi_{\mathrm{I}}, \xi_{\mathrm{II}}, \theta_{\mathrm{I}}-\theta_{\mathrm{II}}\right)=\sigma_{Y}^{2} \exp \left(-\omega^{2} d^{2}\right)
$$

where $\xi_{i}=r_{i} / L$ (with $i=\mathrm{I}$, II) are normalized coordinates, $\sigma_{Y}^{2}$ is the variance of $Y$, $\omega=\sqrt{\pi} L /(2 \lambda), \lambda$ is the correlation length, and $d$ is the Euclidean normalized distance between $\mathbf{r}_{\mathrm{I}}$ and $\mathbf{r}_{\mathrm{II}}$, given by $d=\sqrt{\xi_{\mathrm{I}}^{2}+\xi_{\mathrm{II}}^{2}-2 \xi_{\mathrm{I}} \xi_{\mathrm{II}} \cos \left(\theta_{\mathrm{I}}-\theta_{\mathrm{II}}\right)}$. 


\section{Ensemble moments of particle trajectory}

Since for this type of flow the mean trajectory is radial, we focus only on the trajectory variance. The stochastic differential equation governing the trajectory of a particle in a twodimensional steady-state flow is

$$
\frac{r \mathrm{~d} \varphi}{\mathrm{d} r}=\frac{q_{\theta}(r, \varphi)}{q_{r}(r, \varphi)}
$$

Here $q_{\theta}$ and $q_{r}$ are the tangential and radial components of the specific flux, respectively.

Integrating Eq. (6) with the boundary condition $\varphi\left(r, \mathbf{r}_{0}\right)=\theta_{0}$ yields

$$
\varphi\left(r, \mathbf{r}_{0}\right)=\theta_{0}+\int_{r_{0}}^{r} \frac{q_{\theta}\left(r_{\mathrm{I}}, \varphi_{\mathrm{I}}\right) \mathrm{d} r_{\mathrm{I}}}{q_{r}\left(r_{\mathrm{I}}, \varphi_{\mathrm{I}}\right) r_{I}}
$$

where $\varphi_{\mathrm{I}}=\varphi\left(r_{\mathrm{I}}, \mathbb{r}_{0}\right)$. Eq. (7) provides the transverse position $\varphi$, corresponding to a given radial distance, $r$, of a particle initially located at $\mathbf{r}_{0} \equiv\left(r_{0}, \theta_{0}\right)$ and moving toward the well.

The trajectory variance, representing the variability of the angular position of a particle that has been released at $\xi_{0}=r_{0} / L$ and has reached the radial distance $\xi=r / L$ from the well, can be calculated by making use of the expression for tangential flux covariance developed by
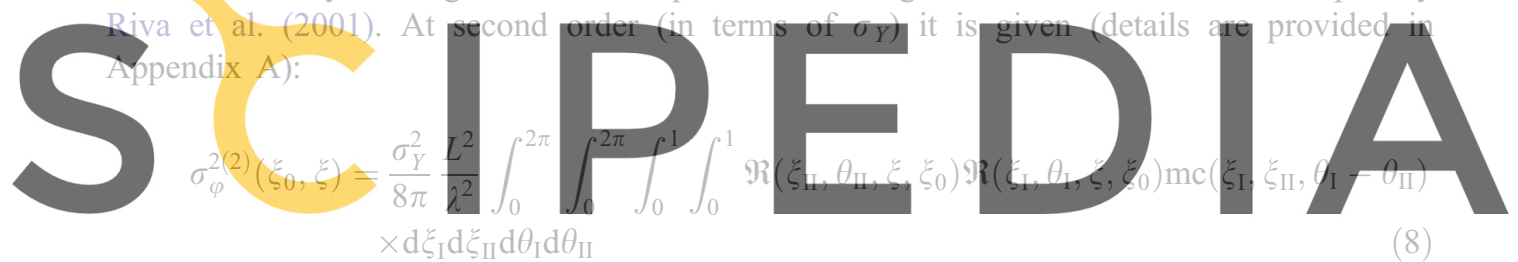

(8)

Register for free at https//www.scipedia.com to download the version without the watermark

Here $\Re\left(\xi_{i}, \theta_{i}, \xi, \xi_{0}\right)(i=\mathrm{I}, \Pi)$ and $\mathrm{mc}\left(\xi_{\mathrm{I}}, \xi_{\mathrm{II}}, \theta_{\mathrm{I}}-\theta_{\mathrm{II}}\right)$ are defined in Appendix B (Eqs. (B8) and (B9), respectively).

The particle trajectory variability is better analyzed by introducing the quantity $\Delta$ defined as:

$$
\Delta\left(r, \mathbf{r}_{0}\right)=r\left|\varphi\left(r, \mathbf{r}_{0}\right)-\left\langle\varphi\left(r, \mathbf{r}_{0}\right)\right\rangle\right|=r\left|\varphi^{\prime}\left(r, \mathbf{r}_{0}\right)\right|
$$

and representing a measure of the deviation of the particle (injected at $\mathbf{r}_{0}$ ) from the mean trajectory, along a circumference arc of radius $r$. Here and in the following angular brackets designate ensemble averaging in probability space and primed quantities are zero-mean random fluctuations about the mean. The second order (in $\sigma_{Y}$ ) variance of $\Delta$ is given by

$$
\sigma_{\Delta}^{2(2)}\left(\xi_{0}, \xi\right)=L^{2} \xi^{2} \sigma_{\varphi}^{2(2)}\left(\xi_{0}, \xi\right)
$$

The analysis of $\sigma_{\Delta}^{2(2)}$ is of more practical use than that of $\sigma_{\varphi}^{2(2)}$, since it would allow demarcating aquifer regions within which a particle initially released at $\xi_{0}$ can be found with a given probability. From Eq. (10) we can find the two limiting values $\sigma_{\Delta}^{2(2)}\left(\xi_{0}, \xi=0\right)=0$ (the length of the arc is zero) and $\sigma_{\Delta}^{2(2)}\left(\xi_{0}, \xi=\xi_{0}\right)=0$ (no uncertainty in the release location).

Gaussian quadratures were employed to evaluate the four-dimensional integrals in Eq. (8). The number of Gauss points needed to attain convergence increases with $L / \lambda$; however, convergence was obtained with less than 80 Gauss points even for the largest $L / \lambda$ values analyzed. 
Fig. 1 illustrates the effect of the particle release position, $\xi_{0}$, on the variability of $\Delta$ by depicting the ratio $\sigma_{\Delta}^{2(2)} /\left(\sigma_{Y}^{2} L^{2}\right)$ calculated on the basis of Eq. (10) as a function of $\xi$ for four release points and $L / \lambda=10$ (Fig. 1a) and 1 (Fig. 1b). The results suggest that the trajectory variance is smallest when particles are released close to the well, since the effects of the deterministic release and ending points are strong. The maximum value of $\sigma_{\Delta}^{2(2)}$ is located between the well and $\xi=\xi_{0} / 2$ so that the curves are (generally) not symmetric. This effect is strong for large correlation scales $(L / \lambda=1)$ while the curves tend to become symmetric (Fig. 1a) as the correlation scale decreases (i.e., $L / \lambda$ increases).

Fig. 2a and $\mathrm{b}$ illustrate the variation of $\sigma_{\Delta}^{2(2)} /\left(\sigma_{Y}^{2} L^{2}\right)$, calculated on the basis of Eq. (10), with $\xi$ and $L / \lambda$, when the particle is released along the domain boundary $\left(\xi_{0}=1\right)$. When $L /$ $\lambda \leq 5, \sigma_{\Delta}^{2(2)}$ generally increases with $L / \lambda$ (see Fig. 2a), except at points close to the well. This is due to two effects: as $L / \lambda$ increases (while keeping $L$ finite) (i) all fields in the ensemble of realizations depart from the homogeneous behavior, where all trajectories are radial (and $\sigma_{\Delta}^{2}=0$ ), and (ii) the effect of the deterministic condition of the release point (that tends to reduce $\sigma_{\Delta}^{2}$ ) decreases. The dependence of $\sigma_{\Delta}^{2(2)}$ on $L / \lambda$ is then reversed when $L / \lambda \geq 6$, i.e., $\sigma_{\Delta}^{2(2)}$ decreases with increasing $L / \lambda$ (see Fig. 2b). This can be explained by analyzing the behavior of $\sigma_{\Delta}^{2(2)}$ for the limiting case $L / \lambda \rightarrow \infty$. The latter can only be obtained by setting $\lambda \rightarrow 0$, since $L$ is finite. This situation might be unrealistic in that it would represent a delta-correlated transmissivity that behaves as a white noise. In this limiting case we obtain that $\lim _{L} \sigma_{4}^{2(2)}=0$ meaning that solute

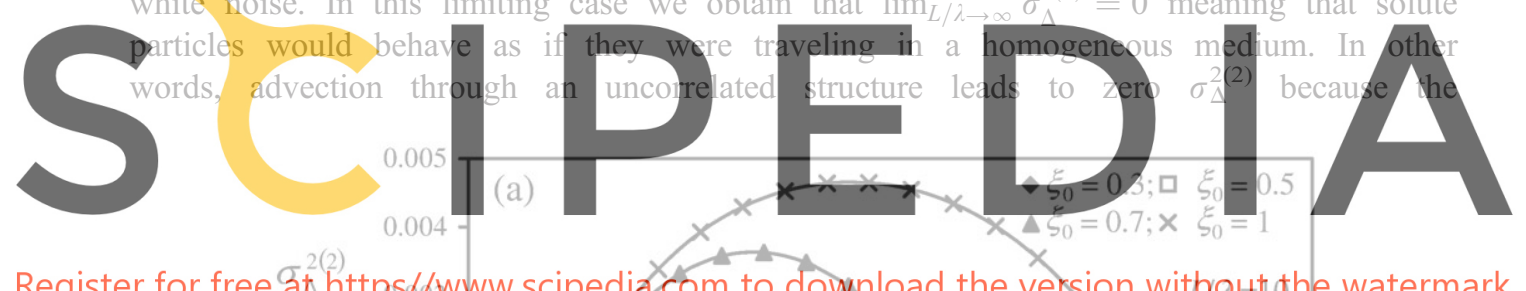

Register for free at httpsos $d$ wwww.scipedia.com to download the vension without the watermark
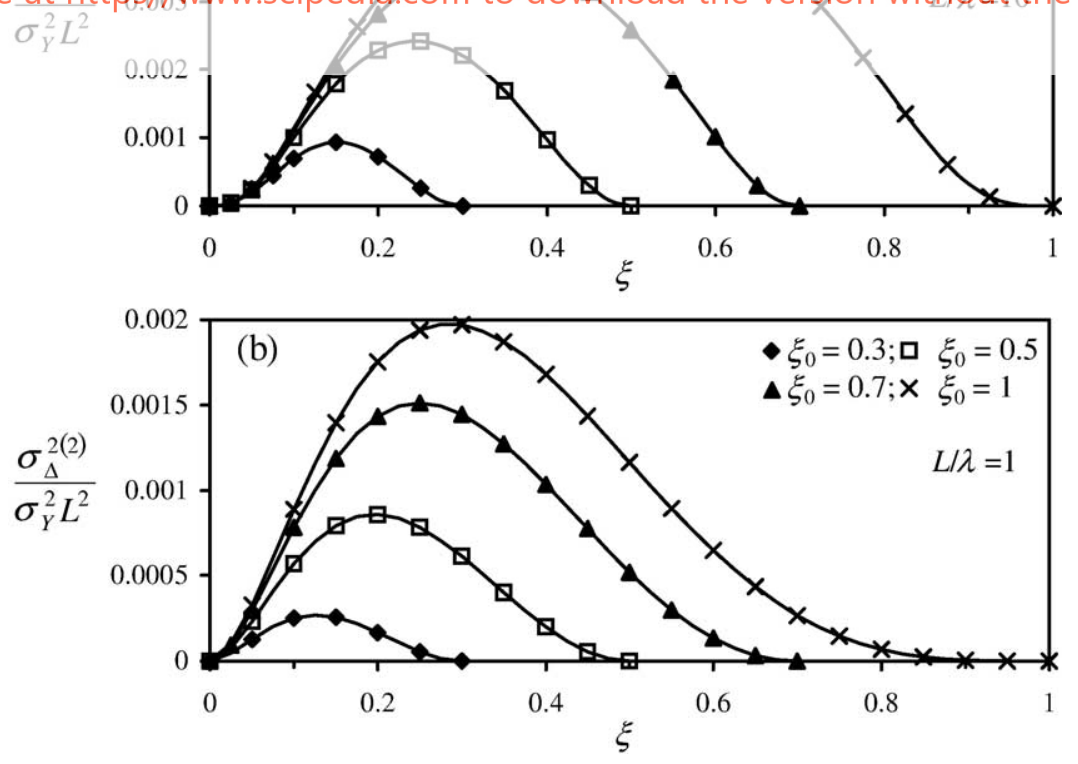

Fig. 1. Normalized variance of particle displacement from the mean trajectory versus normalized distance from the well, $\xi$, for various release locations, $\xi_{0}$, and (a) $L / \lambda=10$; (b) $L / \lambda=1$. 

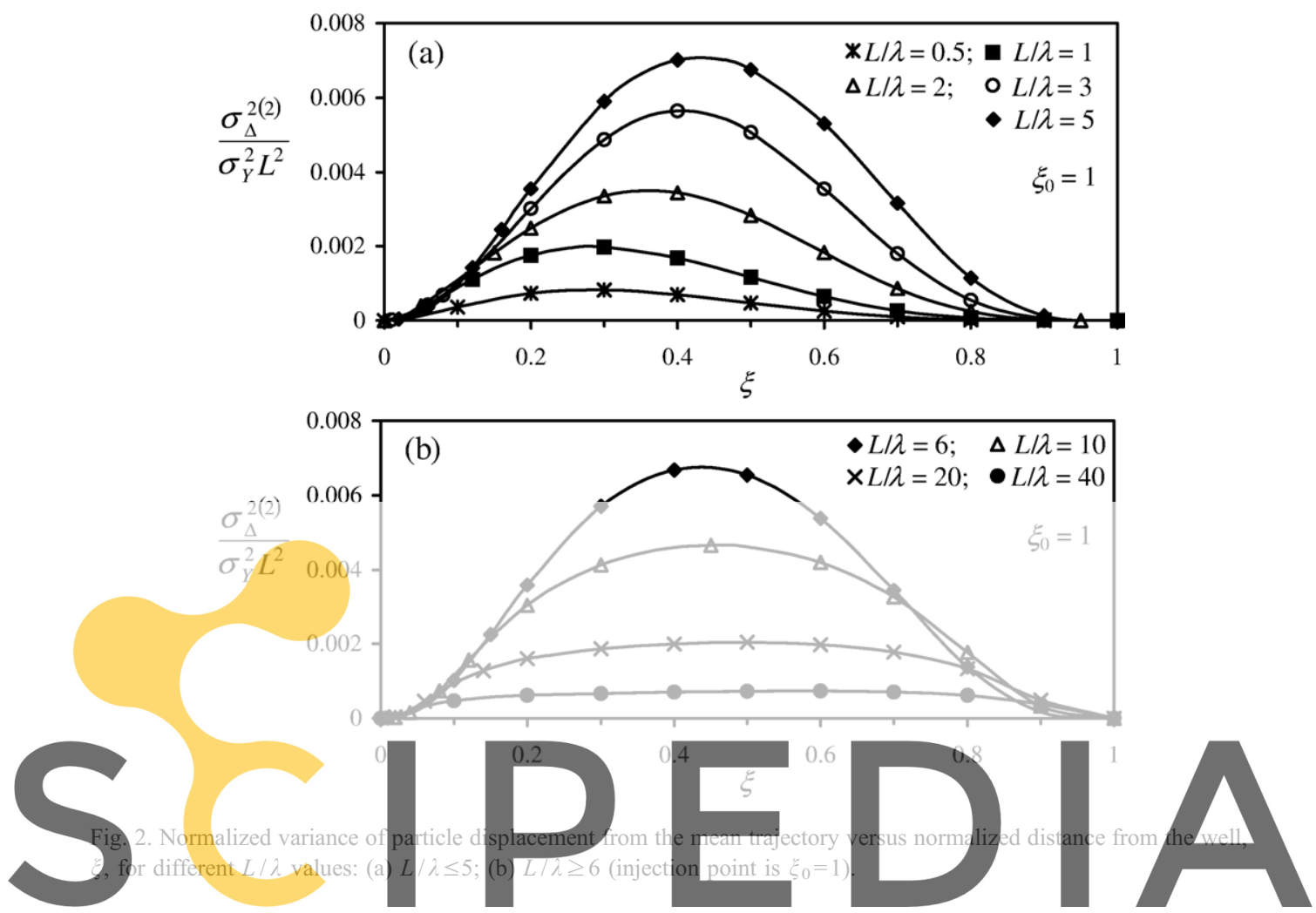

tangential flux is also uncorrelated along the mean trajectory (which is a straight line)

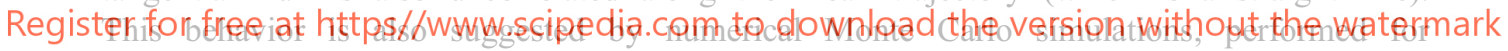

\section{increasing $L / \lambda$ (Section 5).}

Fig. 3 depicts the normalized standard deviation $\sigma_{\varphi}^{2(2)} / \sigma_{Y}$ of the angular particie position versus $L / \lambda$ for four radial positions $(\xi)$ reached by a particle released at $\xi_{0}=1$, based on Eq. (8). The maximum value for $\sigma_{\varphi}^{(2)} / \sigma_{Y}$ is around $30^{\circ}$, and is attained for the largest distance traveled (i.e., in the vicinity of the well) and for $L / \lambda \approx 2$. The curves display (for each $\xi$ ) a local maximum that increases with travel distance (i.e., as $\xi$ decreases).

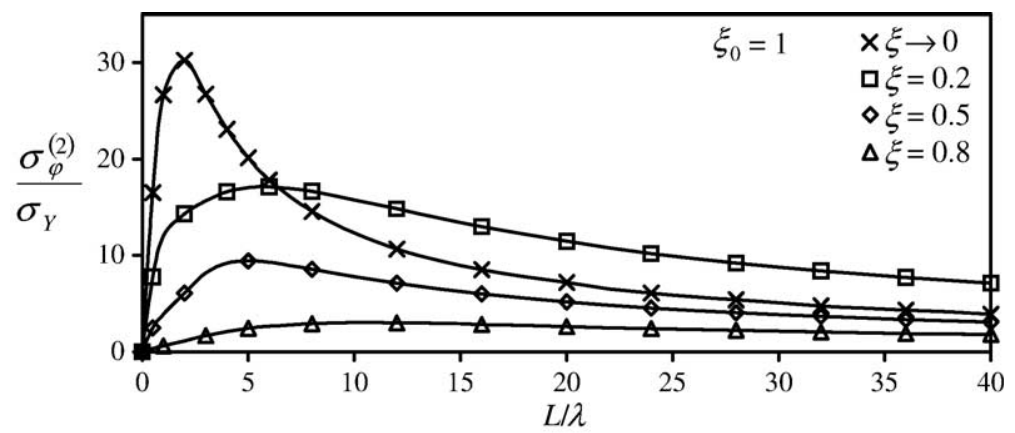

Fig. 3. Normalized standard deviation of the angular particle position (in sexagesimal degrees) versus $L / \lambda$ for different particle locations, $\xi\left(\xi_{0}=1\right)$. 


\section{Ensemble moments of solute travel time}

In the following we provide expressions for the predictor of the travel time, given by its ensemble mean, $\langle t\rangle$, together with travel time variance, $\sigma_{t}^{2}$.

\subsection{Mean travel time}

The starting point is Eq. (4). We follow a procedure similar to that outlined by Guadagnini et al. (2003). Integrating Eq. (4), making use of Reynold's decomposition (decomposing a random quantity, $A$, as the sum of its ensemble mean, $\langle A\rangle$, plus a zero-mean fluctuation, $A^{\prime}$ ), expanding velocities in Taylor's series around the mean trajectory and disregarding terms with powers of fluctuations larger than two, leads to the following expression for the mean travel time that a given particle takes to reach the well:

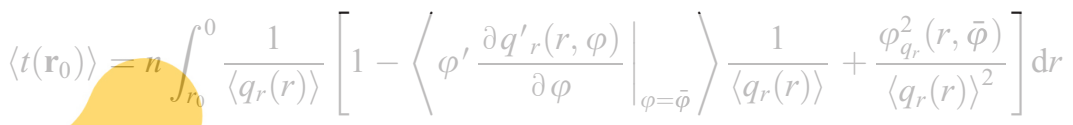

(a) $\left\langle q_{r}(r)\right\rangle$ is the mean radial component of the flux;

(b) $\left\langle\varphi \varphi^{\prime} \frac{\partial q_{r}(r, \varphi)}{\rho r} \mid-\right\rangle$ is the cross-covariance between the trajectory and the tangential derivate
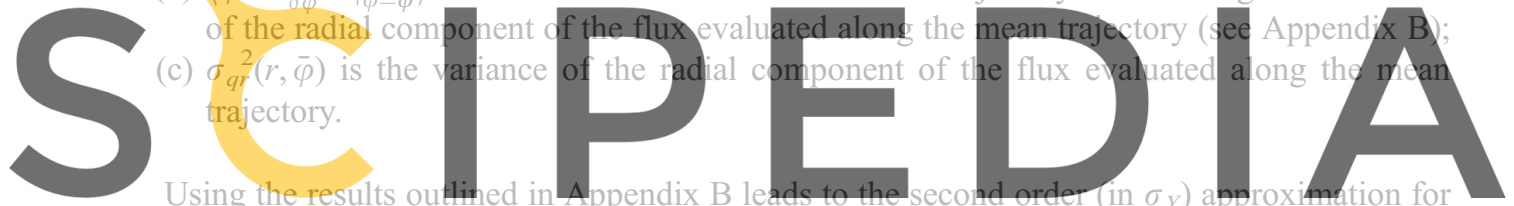

the mean travel time of a particle starting from a deterministic injection point $\left(\xi_{0}=r_{0} / L\right)$. Thus,

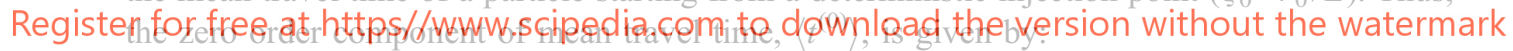

$$
\left\langle t^{(0)}\left(\xi_{0}\right)\right\rangle=n \int_{0}^{\xi_{0}} \frac{2 \pi \xi L^{2}}{Q} \mathrm{~d} \xi=n \frac{\pi L^{2}}{Q} \xi_{0}^{2}
$$

and coincides with the solution for a homogeneous aquifer. The first order component of $\langle t\rangle$, $\left\langle t^{(1)}\right\rangle$, vanishes. Making use of the flux covariance expression developed by Riva et al. (2001) leads to the following expression for the second order (in $\sigma_{Y}$ ) component, $\left\langle t^{(2)}\right\rangle$ :

$$
\left\langle t^{(2)}\left(\xi_{0}\right)\right\rangle=n \frac{8 \pi^{3} L^{4}}{Q^{3}}\left\{\int_{0}^{\xi_{0}} \xi^{2}\left[\left.\int_{\xi}^{\xi_{0}} \frac{\partial C_{q_{r} q \theta}^{(2)}\left(\xi, \xi_{\mathrm{II}}, \varphi, \theta_{0}\right)}{\partial \varphi}\right|_{\varphi=\theta_{0}} \mathrm{~d} \xi_{\mathrm{II}}\right] \mathrm{d} \xi+\int_{0}^{\xi_{0}} \xi^{3} \sigma_{q_{r}}^{2(2)}(\xi) \mathrm{d} \xi\right\} .
$$

Here $C_{q_{r} q_{\theta}}^{(2)}$ and $\sigma_{q_{r}}^{2(2)}$, are the second order cross-covariance between radial and tangential flux components and the variance of the radial flux component, respectively. Since, for this flow scenario, $C_{q_{r} q_{\theta}}^{(2)}$ and $\sigma_{q_{r}}^{2(2)}$ are independent of the angular position (Riva et al., 2001), so is $\left\langle t^{(2)}\right\rangle$. A compact expression for Eq. (13) for the particular case of a Gaussian autocorrelation function for $Y$ is presented in Appendix B (Eq. (B5)). Finally, the second order (in $\sigma_{Y}$ ) approximation, $\left\langle t^{[2]}\right\rangle=\left\langle t^{(0)}\right\rangle+\left\langle t^{(2)}\right\rangle$, of the mean travel time is:

$$
\left\langle t^{[2]}\left(\xi_{0}\right)\right\rangle=n \frac{\pi L^{2}}{Q}\left[\xi_{0}^{2}+\frac{\sigma_{Y}^{2}}{2} \frac{L^{2}}{\lambda^{2}}\left(\mathfrak{I}_{1}+\frac{\mathfrak{I}_{2}}{2 \pi}\right)\right]
$$


where $\mathfrak{I}_{1}$ and $\mathfrak{I}_{2}$ are given by Eqs. (B6) and (B7), respectively. The three- $\left(\mathfrak{I}_{1}\right)$ and five- $\left(\mathfrak{I}_{2}\right)$ dimensional integrals can be evaluated using Gaussian quadratures.

Fig. 4 shows the dependence of the dimensionless second order mean travel time correction, $\left\langle t^{(2)}\right\rangle /\left(\pi n L^{2} \sigma_{Y}^{2} Q^{-1}\right)$, based on Eq. (B5), on $L / \lambda$ for various injection points, $\xi_{0}$. As a limiting case, when $L / \lambda \rightarrow 0$, we obtain $\lim _{L / \lambda \rightarrow 0} t^{(2)}=0$. In this case, the hydraulic conductivity is constant (but unknown), so that in each realization the travel time to the well is given by the zero order solution, with no uncertainty. Fig. 4 reveals that $\left\langle t^{(2)}\right\rangle$ first increases and then, after reaching a maximum, decreases with $L / \lambda$ (note that the decreasing limb of the curves corresponding to injection points located close to the well, i.e., $\xi_{0}=0.1,0.2$, is not shown in Fig. 4). In the limit for $L / \lambda \rightarrow \infty$, we obtain $\lim _{L / \lambda \rightarrow \infty}\left\langle t^{(2)}\right\rangle=0$. The physical explanation of this result is that as $L / \lambda$ increases (while keeping $L$ finite), the particle trajectory (not only its mean value) becomes radial and each realization tends to display a radial symmetry. Therefore, the travel time coincides with $\left\langle t^{(0)}\right\rangle$ for each realization of the ensemble, again showing a similar behavior of the uncorrelated and the homogeneous media. This result is also suggested by numerical Monte Carlo simulations (Section 5).

An important result stemming from Eq. (14) is that $\left\langle t^{(2)}\right\rangle$ is always positive for all cases analyzed, indicating that the actual mean travel time in a two-dimensional randomly heterogeneous domain is always larger than the travel time for a homogeneous medium. As a consequence, using the classical formulae for homogeneous aquifer would always lead to underestimating the mean recovery time. This result can have profound implications in
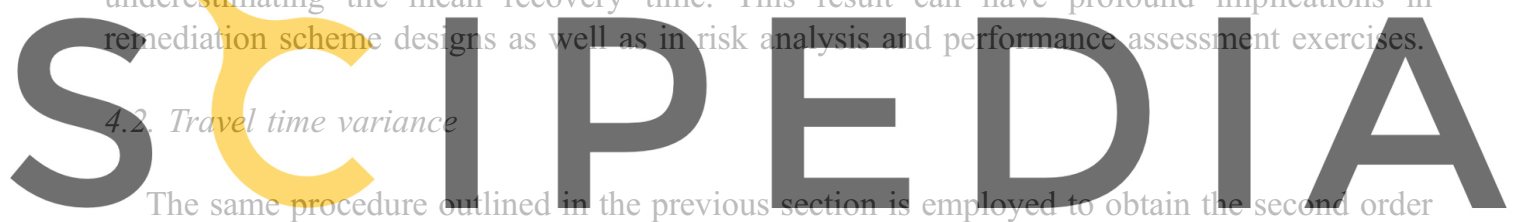

(in $\sigma_{Y}$ ) variance of the travel time to the well, $\sigma_{t}^{2}\left(r_{0}\right)$, of a particle injected at the deterministically Register for frestatahtt.ps//www.scipedia.com to download the version without the watermark

$$
\sigma_{t}^{2(2)}\left(\mathbf{r}_{0}\right)=\frac{16 \pi^{4}}{Q^{4}} n^{2} \int_{r_{0}}^{0} \int_{r_{0}}^{0} C_{q_{r}}^{(2)}\left(r, \bar{\varphi}, r_{\mathrm{I}}, \bar{\varphi}_{\mathrm{I}}\right) r^{2} r_{\mathrm{I}}^{2} \mathrm{~d} r \mathrm{~d} r_{\mathrm{I}}
$$

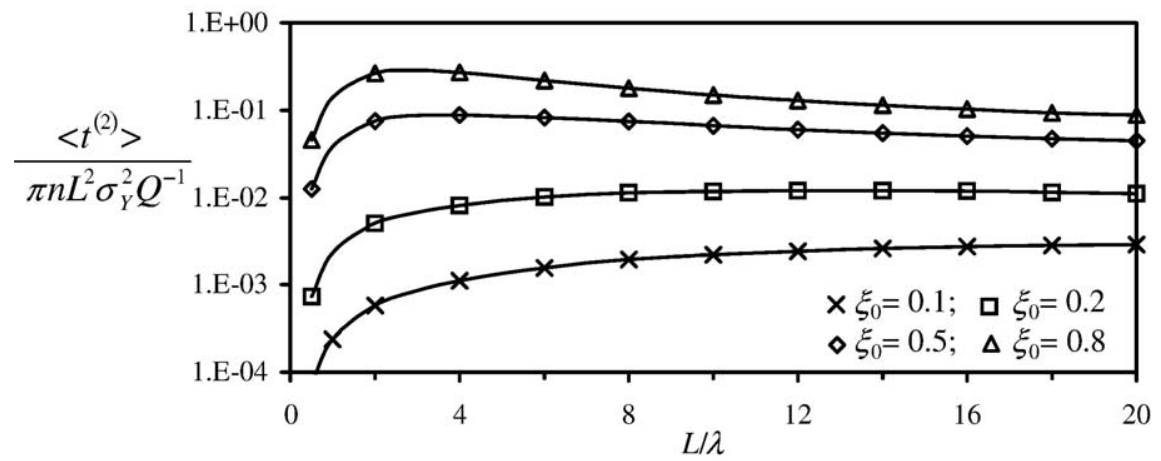

Fig. 4. Normalized second order correction of mean travel time versus $L / \lambda$ for various particle injection locations, $\xi_{0}$. 


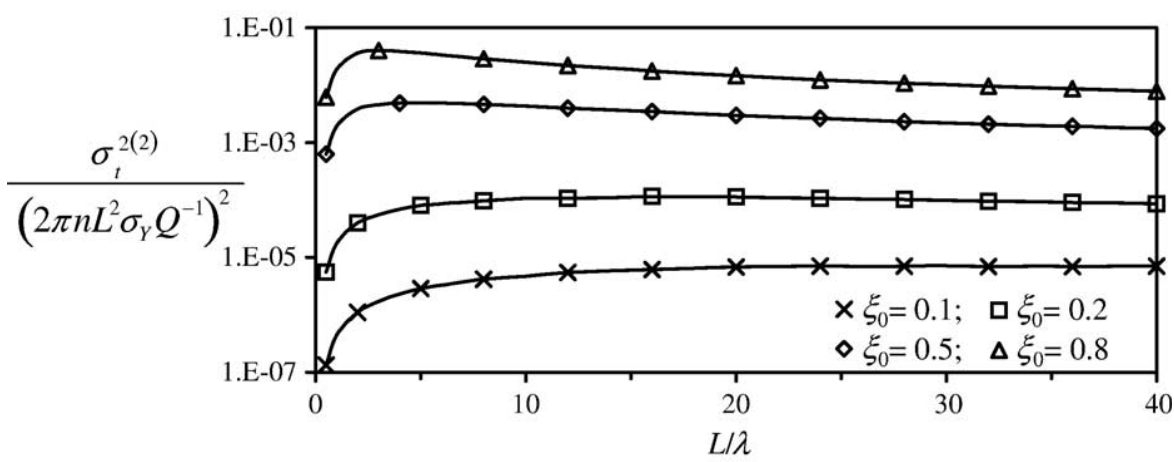

Fig. 5. Normalized travel time variance versus $L / \lambda$ for various particle injection locations, $\xi_{0}$.

where $C_{q_{r}}^{(2)}$ is the (second order) covariance of the radial flux evaluated at two radial distances from the well along the mean trajectory. For a Gaussian autocorrelation function of $Y$, the travel time variance (in dimensionless coordinates) reduces to

$$
\begin{aligned}
\sigma_{t}^{2(2)}\left(\xi_{0}\right)= & 4 \pi^{2} n^{2} \frac{L^{4}}{Q^{2}} \sigma_{Y}^{2}\left\{\frac{2}{3 \pi} \frac{\lambda^{2}}{L^{2}}\left(2 \exp \left[-\omega^{2} \xi_{0}^{2}\right]\left(\xi_{0}^{2}-\frac{1}{2 \omega^{2}}\right)+\frac{1}{\omega^{2}}-3 \xi_{0}^{2}\right)\right. \\
& \left.+\frac{2}{3} \frac{\lambda}{L} \xi_{0}^{3} \operatorname{erf}\left[\omega \xi_{0}\right]-\frac{\xi_{0}^{4}}{4}+\frac{1}{\pi}\left(\frac{1}{8} \frac{L^{2}}{\lambda^{2}} \mathfrak{I}_{3}+\mathfrak{I}_{4}\right)\right\} .
\end{aligned}
$$

The multidimensional integrals, $\mathfrak{I}_{3}$ and $\mathfrak{I}_{4}$, are defined in Appendix C. Similarly to $\left\langle t^{(2)}\right\rangle$, the travel time variance is independent of $\theta_{0}$.

When the release point is close to the well $\left(\xi_{0} \rightarrow 0\right)$, Eq. (16) reduces to the obvious solution $\sigma_{t}^{2(2)} \rightarrow 0$. For the non-trivial case, one is also interested in the limiting values. First, for very large $\lambda(L / \lambda \rightarrow 0)$, we have $\lim _{L / \lambda \rightarrow 0} \sigma_{t}^{2(2)}=0$, independently of the release location, as there is no uncertainty in the travel time. Second, in the limiting case of an uncorrelated medium (i.e., $L / \lambda \rightarrow \infty$, while keeping $L$ finite), we have $\lim _{L / \lambda \rightarrow \infty} \sigma_{t}^{2(2)}=0$, consistently with what noted in the previous sections regarding delta-correlated fields.

Fig. 5 shows how the dimensionless quantity $\sigma_{t}^{2(2)} /\left(2 \pi n L^{2} \sigma_{Y} Q^{-1}\right)^{2}$, based on Eq. (16), varies with $L / \lambda$ for various injection points, $\xi_{0}$. As above, Gaussian quadratures have been employed to evaluate the two- $\left(\mathfrak{I}_{4}\right)$ and four- $\left(\mathfrak{I}_{3}\right)$ dimensional integrals in Eq. (16). In agreement with the Monte Carlo-based numerical solutions obtained by Riva et al. (1999), the variance of travel time increases monotonically with travel distance. With regard to the relationship between $\sigma_{t}^{2(2)}$ and $L / \lambda$, the behavior is qualitatively identical as that of the correction term to the mean travel time, $\left\langle t^{(2)}\right\rangle$.

\section{Numerical demonstration of the analytical second order solution}

The accuracy of our analytical second order solutions was checked against numerical Monte Carlo simulations. The latter were performed by using the same code of Riva et al. (1999), with different boundary conditions. Flow and transport are simulated in a square domain with 100 rows and 100 columns of uniform size $(\Delta x=\Delta y=\delta)$. A circular boundary of radius $L=50 \delta$ was defined around the well by designating all cells outside it as inactive.

The hydraulic head along the circular boundary was set constant. A well pumping at the constant rate $Q=100$ (in consistent units) was placed at the central node of the grid. A Gaussian 
sequential simulator code (GCOSIM3D, Gómez-Hernández, 1991), was used to generate random realizations of $Y$ on the above defined two-dimensional grid. Each realization constituted a sample from a multivariate Gaussian, statistically homogeneous field, with ensemble mean $\langle Y\rangle=0$ and an isotropic Gaussian covariance function. Simulations were performed by varying the main parameters within the following ranges: $0.1 \leq \sigma_{Y}^{2} \leq 1.0$, and $1 \leq L / \lambda \leq 10$. The effective porosity, $n$, is taken equal to 0.3 . Flow is solved by Galerkin finite elements using bilinear shape functions. Solute transport in each realization is modeled by Particle Tracking, according to the following procedure. An ideal tracer particle is located at each grid node along 4 perpendicular axes centered at the well and its purely advective movement is modeled by

$$
\mathbf{r}^{m+1}=\mathbf{r}^{m}+V\left(\mathbf{r}^{m}\right) \Delta s /\left|\mathbf{V}\left(\mathbf{r}^{m}\right)\right| .
$$

Here $\mathbf{r}^{m+1}$ and $\mathbf{r}^{m}$ are the particle locations at times $(m+1)$ and $m$, respectively; $\mathbf{V}\left(\mathbf{r}^{m}\right)$ is the seepage velocity computed at $\mathbf{r}^{m}$ and $\left|\mathbf{V}\left(\mathbf{r}^{m}\right)\right|$ is its module. The maximum displacement $\Delta s=\delta /$ 1000 to be used in Eq. (17) was chosen empirically by adjusting it until an acceptable compromise between simulation time and numerical accuracy in the reproduction of the particle trajectory was obtained (for additional details, see Guadagnini and Franzetti, 1999).

The number of Monte Carlo simulations needed to obtain stability of travel time and trajectory moments increases with the order of the statistical moment studied and with $\sigma_{Y}^{2}$, and (in general) decreases with increasing $L / \lambda$. An acceptable compromise between CPU time requirements and accuracy of reproduction of the analyzed statistical moments was obtained
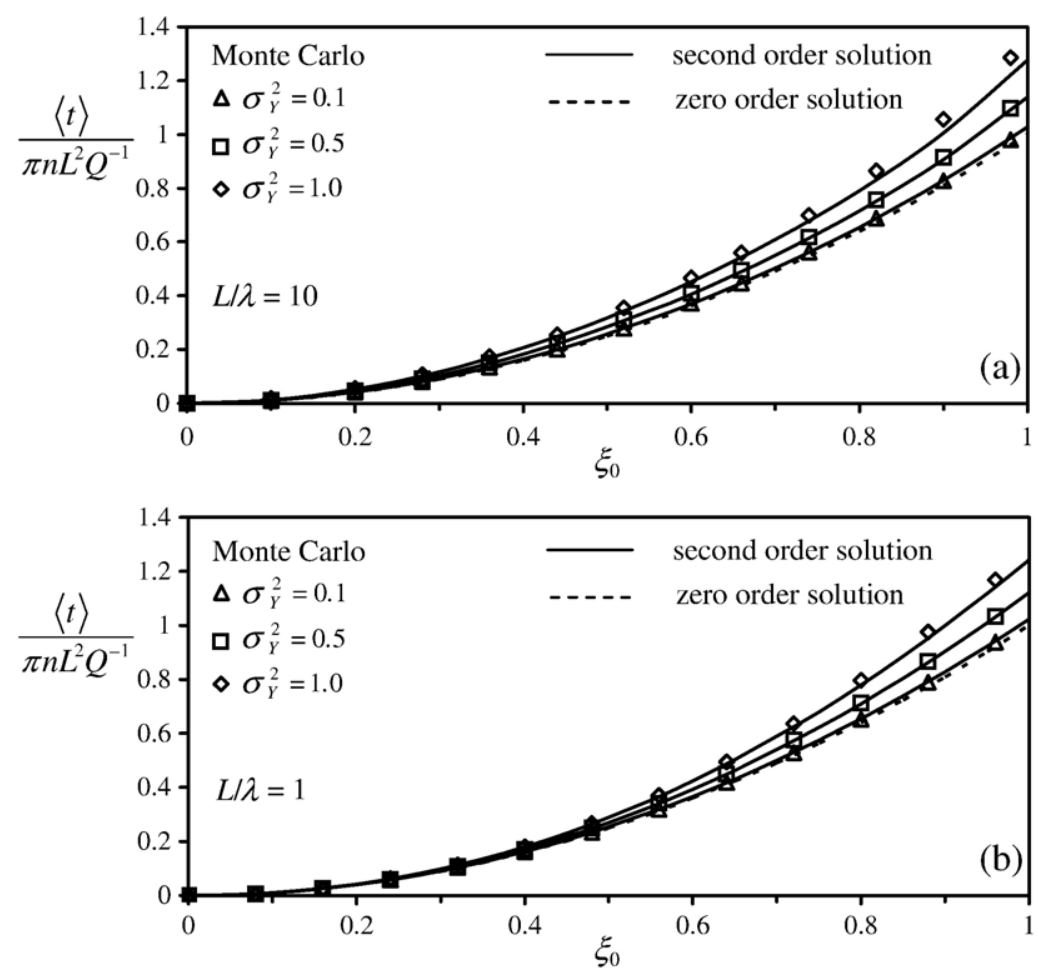

Fig. 6. Comparison between zero and second order analytical solutions and numerical Monte Carlo simulations of the normalized mean travel time as a function of the particle injection location, $\xi_{0}$, and $\sigma_{Y}^{2}$ : (a) $L / \lambda=10 ;$ (b) $L / \lambda=1$. 
with 4000 Monte Carlo iterations. The assessment of the reliability of the inferred computed moments was performed both qualitatively and quantitatively on the basis of simple rules of statistical inference (Ballio and Guadagnini, 2004). The maximum computed difference associated to a probability level of 0.95 between the sample and the target (i.e., evaluated with an infinite number of Monte Carlo iterations) statistical moments is equal to $0.031 \sigma$ and $0.045 \sigma^{2}$, respectively for the first and second order statistical moments. Here $\sigma$ represents the standard deviation of the variable of interest (either travel time or trajectory).

Fig. $6 \mathrm{a}$ and $\mathrm{b}$ depict the dimensionless travel time corresponding to different values of $\sigma_{Y}^{2}$, as obtained by zero and second order analytical solutions (Eqs. (12) and (14)) and by Monte Carlo simulations, with $L / \lambda=10$ and $L / \lambda=1$, respectively. The effect of the second order travel time correction, $\left\langle t^{(2)}\right\rangle$, becomes more significant as $\sigma_{Y}^{2}$ and $\xi_{0}$ increase. The agreement between analytical and Monte Carlo solutions is excellent for $\sigma_{Y}^{2}<1$ and deteriorates as $\sigma_{Y}^{2}$ increases, consistently with the order of the perturbation expansion.

Fig. $7 \mathrm{a}$ and $\mathrm{b}$ illustrate the comparison between the dimensionless travel time variance for three values of $\sigma_{Y}^{2}(=0.1 ; 0.5 ; 1.0)$, as obtained by our second order analytical solution (Eq. (16)) and Monte Carlo simulations with $L / \lambda=10$ and $L / \lambda=1$, respectively. With the only exception of the case $\sigma_{Y}^{2}=0.1$, the analytical solution consistently underestimates the travel time variance. This suggests that the travel time variance displays a supra-linear increase with $\sigma_{Y}^{2}$, so that its proper representation in highly heterogeneous aquifers under radial flow conditions apparently
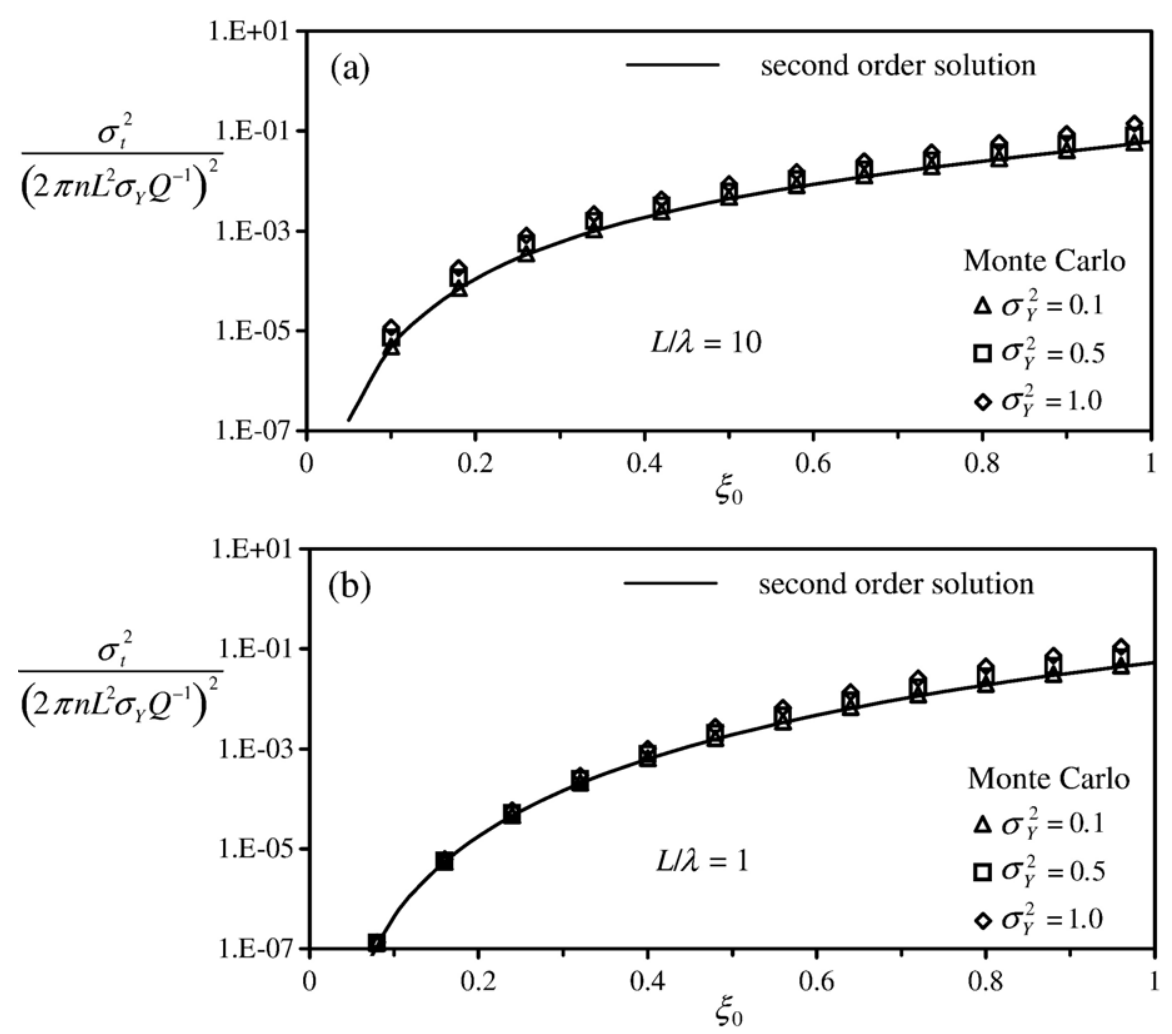

Fig. 7. Comparison between second order analytical solutions and numerical Monte Carlo simulations of the normalized travel time variance as a function of the particle injection location, $\xi_{0}$, and $\sigma_{Y}^{2}$ : (a) $L / \lambda=10$; (b) $L / \lambda=1$. 


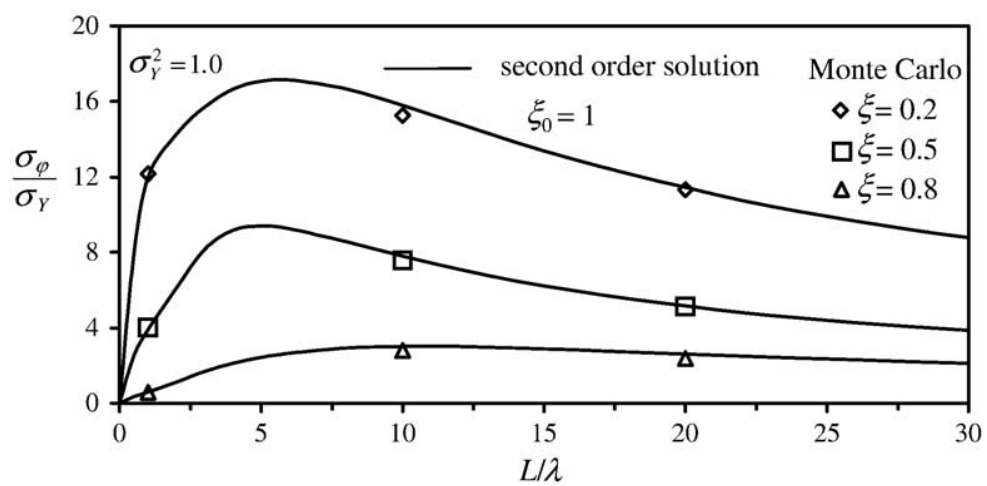

Fig. 8. Comparison between analytical and Monte Carlo normalized standard deviation of the angular particle position (in sexagesimal degrees) for $L / \lambda=1,10,20$ and different particle locations, $\xi\left(\xi_{0}=1\right)$. Numerical Monte Carlo results are computed for $\sigma_{Y}^{2}=1$.
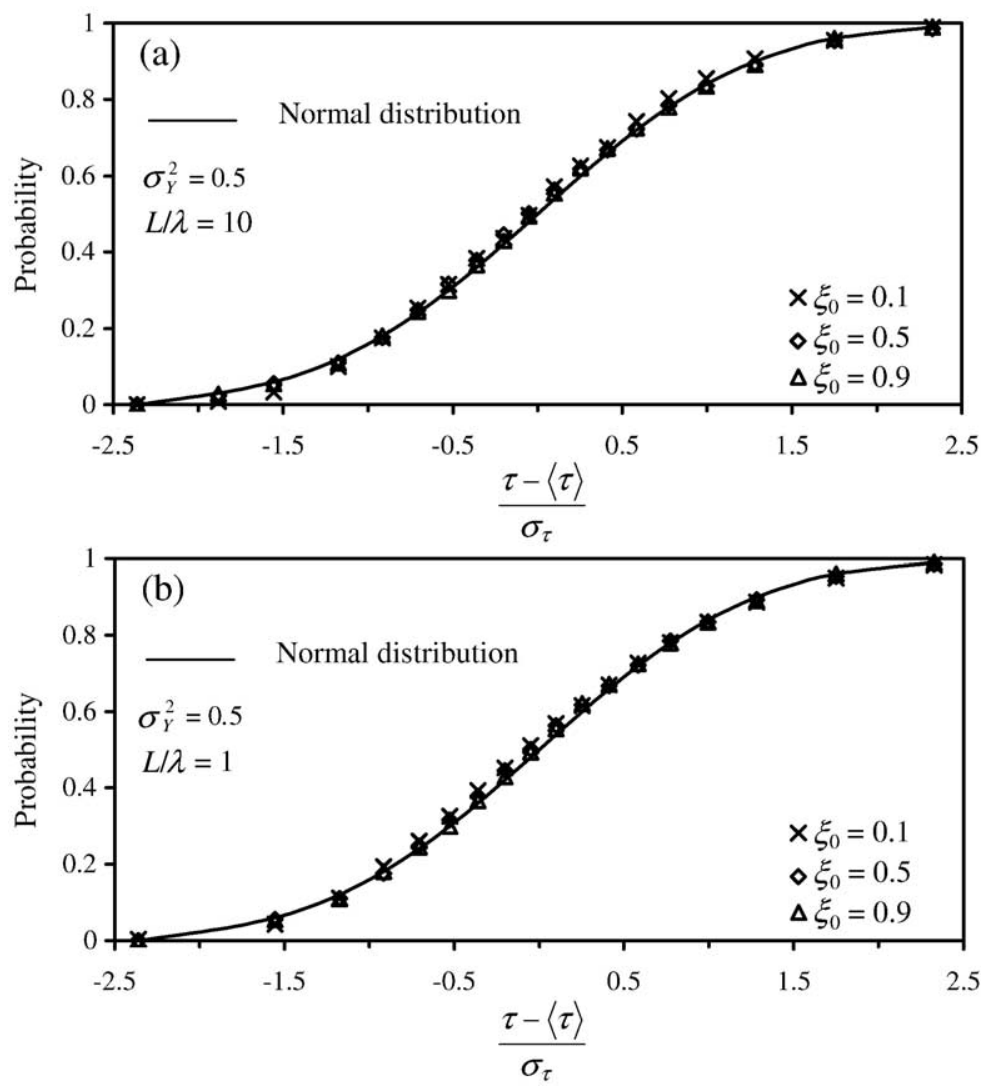

Fig. 9. Comparison between the Normal distribution and the Monte Carlo cumulative distribution of the natural logarithm of travel time for various injection locations, $\xi_{0}$, and $\sigma_{Y}^{2}$ : (a) $L / \lambda=10$; (b) $L / \lambda=1$. 
requires developing higher order approximations, with an increase in mathematical complexity and computational burden. For the same set of parameters, the agreement between the analytical dimensionless displacement variance, $\sigma_{\Delta}^{2(2)} /\left(\sigma_{Y}^{2} L^{2}\right)$, and its Monte Carlo based counterparts is satisfactory (not shown).

Fig. 8 compares the analytical results of Fig. 3 against numerical Monte Carlo simulations, calculated for $\sigma_{Y}^{2}=1.0$ and $L / \lambda=1,10,20$, as a support to the analysis presented in Section 3 regarding the limiting behavior of $\sigma_{\varphi}^{(2)}$ for very large $L / \lambda$ (while keeping $L$ finite).

For weakly heterogeneous fields $\left(\sigma_{Y}^{2}=0.1\right)$ chi-square tests of the null hypothesis that the natural logarithm of travel times, $\tau=\ln t$, generated by the Monte Carlo method is Gaussian were positive at a significance level of 5\% for all particle starting positions. However, as $\sigma_{Y}^{2}$ increases, $\tau$ passes the normality test only for starting locations far from the well $\left(\xi_{0}>0.5\right)$, even though the visual agreement with the Normal distribution appears satisfactory for all $\xi_{0}$. An example of the results obtained is shown in Fig. 9a and $\mathrm{b}$ for $\sigma_{Y}^{2}=0.5$ and $L / \lambda=10$ and $L / \lambda=1$, respectively.

Chi-square tests of the null hypothesis that either the trajectories or their natural logarithms are Gaussian were negative at a significance level of 5\%, even though, for practical purposes it can be noted that $\varphi$ displays a good visual agreement with the Normal distribution, as shown, for example, in Fig. 10a and $\mathrm{b}$ for $\sigma_{Y}^{2}=0.5$ and $L / \lambda=10$ and $L / \lambda=1$, respectively.
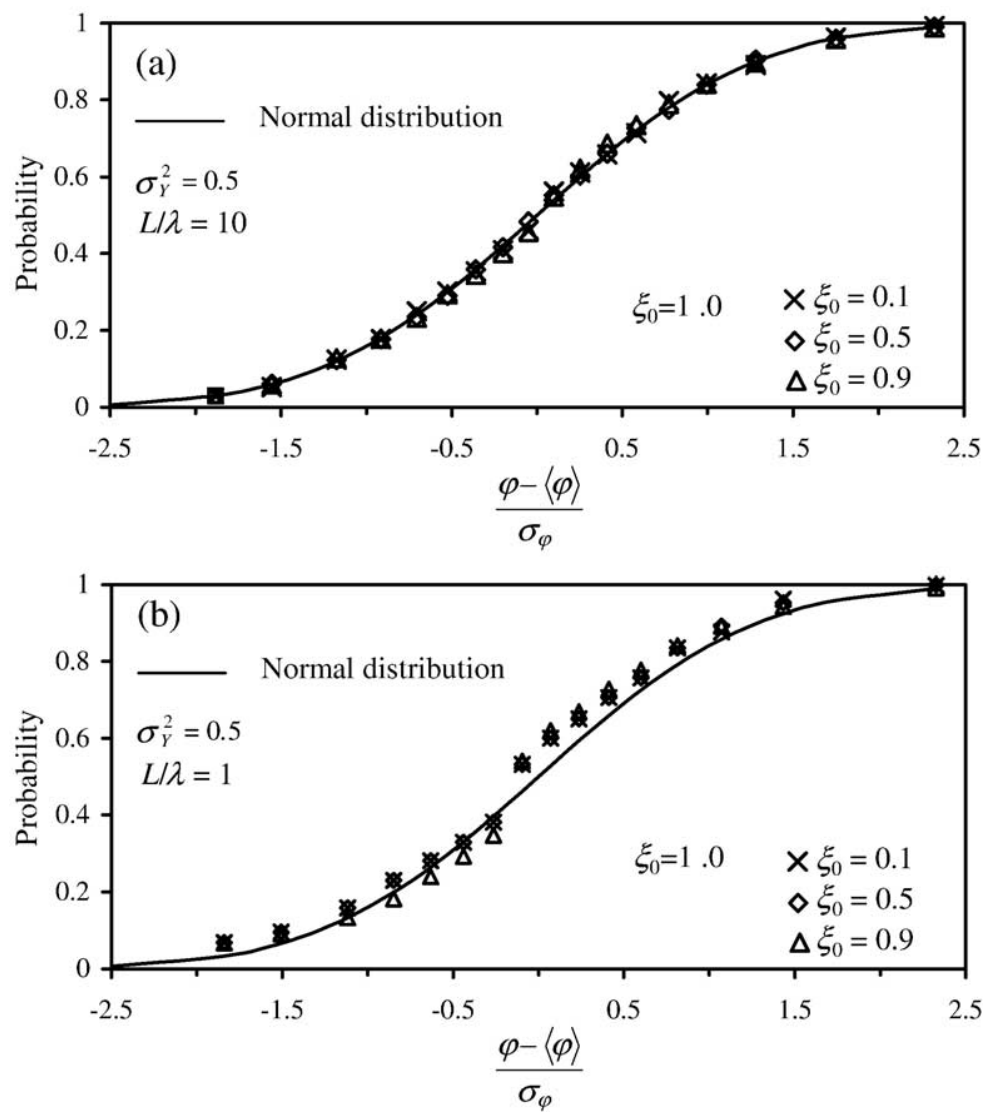

Fig. 10. Comparison between the Normal distribution and the Monte Carlo cumulative distribution of the angular particle displacement for various injection locations, $\xi_{0}$, and $\sigma_{Y}^{2}$ : (a) $L / \lambda=10$; (b) $L / \lambda=1$. 
Assuming log-Gaussianity in the probability distribution of travel time and trajectories would now allow the use of the results here presented in performance assessment or risk analyses practice. For example, regarding travel times it would be possible to obtain the time corresponding to "first arrival", defined as the time for a given percentile of solute to reach the well, or apply a transfer function to relate the probability distribution of travel time to a probability distribution of risk. Regarding trajectories it would be possible to define the potential extension of some areas with a given probability of containing the solutes released, for instance, from a waste repository site.

\section{Conclusions}

We provide original formal expressions for the second order (in $\sigma_{Y}$ ) statistical moments (mean and variance) of travel time and trajectory of conservative solutes advected under twodimensional convergent flow conditions in randomly heterogeneous porous media. Assuming that the parameters defining mass transfer processes between a mobile and an immobile zone are homogeneous, the knowledge of mean and variance of travel time and trajectory of a conservative solute allows obtaining the statistical moments characterizing displacement of a sorptive solute. All the (ensemble) moments presented are given in integral format yielding their explicit dependence on the statistical property of the log-conductivity field. The analytical integral expressions are evaluated using Gaussian quadratures. The seemingly formal complexity of the expressions presented is a consequence of the difficulty inherent to the problem examined. Nevertheless, it allows obtaining valuable insight on the nature of the solution.

The following major conclusions are drawn from the paper.

(1) Valuable information about the variance of the trajectory can be derived by the study of the deviation of the particle from its mean trajectory along an arc, $\Delta$. The study of $\sigma_{\Delta}^{2}$ can be of practical use since it allows demarcating the domain region where contamination of a known source can be found. This variance is zero both at the injection point and at the well, and it displays a maximum whose value and location depend upon the initial injection point, $\sigma_{\Delta}^{2}$ and $L / \lambda$. Since our results give an answer to the basic question of the extent of the lateral spreading of solutes at a given radial distance from a pumping well, a natural extension would include examining the trajectory variance along the radial direction, rendering the uncertainty related to the radial location of a particle at a given time-of-travel.

(2) Even for conservative tracers, the mean travel time for a heterogeneous domain is always larger than that calculated for a homogeneous medium, since it can be expressed (to second order in $\sigma_{Y}^{2}$ ) as the sum of a term corresponding to the travel time in a homogeneous domain, $\left\langle t^{(0)}\right\rangle$, and a positive correction, $\left\langle t^{(2)}\right\rangle$. The latter increases with the radial distance from the well and the degree of heterogeneity of the aquifer (as rendered by $\sigma_{Y}^{2}$ ). It also has a non-monotonic dependence on $L / \lambda$, so that $\left\langle t^{(2)}\right\rangle$ vanishes when the domain is either very small or very large (with respect to $\lambda$ ). In these two limiting cases the mean travel time (at least at second order in $\sigma_{Y}$ ) coincides with that obtained in a homogeneous aquifer.

(3) The second order approximation of the travel time variance, $\sigma_{t}^{2(2)}$, increases monotonically with the distance between the release point and the well, and is a function of $\sigma_{Y}^{2}$ and $L / \lambda$. For small values of $L / \lambda, \sigma_{t}^{2(2)}$ increases with $L / \lambda$. Then the dependence of $\sigma_{t}^{2(2)}$ on $L / \lambda$ is reversed and $\sigma_{t}^{2(2)}$ decreases as $L / \lambda$ increases, while keeping $L$ finite.

(4) The natural logarithm of travel time passes the Gaussianity test for the cases considered. As low order approximations of both the mean and variance have been provided, the full 
pdf of travel time is available. This knowledge can be used in aquifer remediation projects, as it can be used to provide confidence intervals about the time needed for a conservative or sorbing solute particle to reach the pumping well. Another immediate application would be that of extending the work to solute fluxes, which would be of interest in risk analysis and performance assessment exercises.

\section{Acknowledgments}

This work was partly supported by the European Commission under Contract No. EVK1-CT1999-00041 (W-SAHaRA Stochastic Analysis of Well Head Protection and Risk Assessment), and by ENRESA, and by the Departament d'Universitats, Recerca i Societat de la Informació of the Catalan Government.

\section{Appendix A. Trajectory variance}

Multiplying Eq. (6) by the fluctuation, $\varphi^{* \prime}=\varphi^{\prime}\left(r^{*}, \mathbf{r}_{0}\right)$, of the trajectory at the point of radial coordinate $r^{*}$, and taking expectation yields a differential equation satisfied by the covariance of the angular positions respectively reached by a particle at radial coordinates $r$ and $r^{*}, C_{\varphi}\left(r, r^{*}, \mathbf{r}_{0}\right)=\left\langle\varphi^{\prime} \varphi^{* \prime}\right\rangle$ :

$$
\frac{r \mathrm{~d} C_{\varphi}\left(r, r^{*}, \mathbf{r}_{0}\right)}{\mathrm{d} r}=\left\langle\varphi^{* \prime} \frac{q_{\theta}(r, \varphi)}{q_{r}(r, \varphi)}\right\rangle
$$

with boundary condition $C_{\varphi}\left(r, r^{*}, \mathbf{r}_{0}\right)=C_{\varphi}^{0}$ (at $\left.r \equiv r_{0}\right)$. When the initial position is deterministically known $\left(\theta_{0}^{\prime}=0\right)$, then $C_{\varphi}^{0}=0$. Expanding $q_{r}$ and $q_{\theta}$ around the mean trajectory, $\bar{\varphi}=\left\langle\varphi\left(r, \mathbf{r}_{0}\right)\right\rangle$, and integrating Eq. (A1) with deterministic boundary conditions (i.e., $C_{\varphi}^{0}=0$ ), yields (at second order in $\sigma_{Y}$ )

$$
C_{\varphi}^{(2)}\left(r, r^{*}, \mathbf{r}_{0}\right)=-\frac{2 \pi}{Q} \int_{r_{0}}^{r}\left\langle\varphi^{* \prime} q_{\theta}^{\prime}\left(r_{\mathrm{I}}, \bar{\varphi}_{\mathrm{I}}\right)\right\rangle^{(2)} \mathrm{d} r_{\mathrm{I}} .
$$

The trajectory variance is obtained from the covariance in the limit for $r^{*} \rightarrow r$,

$$
\sigma_{\varphi}^{2(2)}\left(r, \mathbf{r}_{0}\right)=-\frac{2 \pi}{Q} \int_{r_{0}}^{r}\left\langle\varphi^{\prime} q_{\theta}^{\prime}\left(r_{\mathrm{I}}, \bar{\varphi}_{\mathrm{I}}\right)\right\rangle^{(2)} \mathrm{d} r_{\mathrm{I}} .
$$

The trajectory variance is therefore a function of the cross-moment between $\varphi\left(r, \mathbf{r}_{0}\right)$ and the tangential component of Darcy's velocity. To obtain this cross-moment we multiply Eq. (6) by the flux residual $q_{\theta}^{\prime}\left(r_{\mathrm{I}}, \bar{\varphi}_{\mathrm{I}}\right)$ and take ensemble average:

$$
\left\langle r \frac{\mathrm{d} \varphi}{\mathrm{d} r} q_{\theta}^{\prime}\left(r_{\mathrm{I}}, \bar{\varphi}_{\mathrm{I}}\right)\right\rangle=\left\langle\frac{q_{\theta}(r, \varphi)}{q_{r}(r, \varphi)} q_{\theta}^{\prime}\left(r_{\mathrm{I}}, \bar{\varphi}_{\mathrm{I}}\right)\right\rangle .
$$

Expanding $q_{r}$ and $q_{\theta}$ around the mean trajectory, the second order (in $\sigma_{Y}$ ) cross covariance between the trajectory evaluated at the radial position $r$ and the tangential component of Darcy's velocity evaluated at $r_{\mathrm{I}}$ along the mean trajectory, $C_{\varphi q \theta}^{(2)}\left(r, r_{\mathrm{I}}, \mathbf{r}_{0}\right)=\left\langle\varphi^{\prime} q_{\theta}^{\prime}\left(r_{\mathrm{I}}, \bar{\varphi}_{\mathrm{I}}\right)\right\rangle^{(2)}$, satisfies the following equation:

$$
\frac{\mathrm{d} C_{\varphi q \theta}^{(2)}\left(r, r_{\mathrm{I}}, \mathbf{r}_{0}\right)}{\mathrm{d} r}=-\frac{2 \pi}{Q} C_{q \theta}^{(2)}\left(r_{\mathrm{I}}, \bar{\varphi}_{\mathrm{I}}, r, \bar{\varphi}\right) .
$$


Here $C_{q \theta}^{(2)}\left(r_{\mathrm{I}}, \bar{\varphi}_{\mathrm{I}}, r, \bar{\varphi}\right)$ is the $O\left(\sigma_{Y}^{2}\right)$ approximation of the covariance of the tangential component of the velocity, evaluated along the mean trajectory at the radial coordinates $r_{\mathrm{I}}$ and $r$. The corresponding boundary condition that applies to Eq. (A5) is $C_{\varphi q \theta}^{(2)}\left(r, r_{\mathrm{I}}, \mathbf{r}_{0}\right)=C_{\varphi q \theta}^{0}$ (at $\left.r \equiv r_{0}\right), C_{\varphi q \theta}^{0}$ being the covariance between the angular position of the injection point and the tangential component of Darcy's velocity evaluated along the mean trajectory at the radial coordinate $r_{\mathrm{I}}$. When the location $\mathbf{r}_{0}$ is deterministically known (i.e., $\theta_{0}^{\prime}=0$ ), then $C_{\varphi q \theta}^{0}=0$. Assuming deterministic boundary conditions, integration of Eq. (A5) yields:

$$
C_{\varphi q \theta}^{(2)}\left(r, r_{\mathrm{I}}, \mathbf{r}_{0}\right)=-\frac{2 \pi}{Q} \int_{r_{0}}^{r} C_{q \theta}^{(2)}\left(r_{\mathrm{I}}, \bar{\varphi}_{\mathrm{I}}, r_{\mathrm{II}}, \bar{\varphi}_{\mathrm{II}}\right) \mathrm{d} r_{\mathrm{II}}
$$

Finally, substituting Eq. (A6) into Eq. (A3) leads directly to

$$
\sigma_{\varphi}^{2(2)}\left(r, \mathbf{r}_{0}\right)=\frac{4 \pi^{2}}{Q^{2}} \int_{r_{0}}^{r} \int_{r_{0}}^{r} C_{q \theta}^{(2)}\left(r_{\mathrm{I}}, \bar{\varphi}_{\mathrm{I}}=\theta_{0}, r_{\mathrm{II}}, \bar{\varphi}_{\mathrm{II}}=\theta_{0}\right) \mathrm{d} r_{\mathrm{I}} \mathrm{d} r_{\mathrm{II}} .
$$

Riva et al. (2001) show that for convergent flow the tangential flux covariance between two points $\left(r_{\mathrm{I}}, \theta_{\mathrm{I}}\right)$ and $\left(r_{\mathrm{II}}, \theta_{\mathrm{II}}\right)$ depends only on angular separation, that is $C_{q \theta}^{(2)}\left(r_{\mathrm{I}}, \theta_{\mathrm{I}}\right.$, $\left.r_{\mathrm{II}}, \theta_{\mathrm{II}}\right)=C_{q \theta}^{(2)}\left(r_{\mathrm{I}}, r_{\mathrm{II}}, \theta_{\mathrm{I}}-\theta_{\mathrm{II}}\right)$. Thus $\sigma_{\varphi}^{2(2)}$ is independent of the release point angular position. Introducing the dimensionless coordinate $\xi=r / L$ and using the expression for $C_{q \theta}^{(2)}$ provided by Riva et al. (2001), leads directly to Eq. (8), expressing the second order component of the trajectory variance of a particle starting at $\xi_{0}$ and reaching radial location $\xi$.

A necessary check to the solution is given by the following limiting cases. First, when the domain is very small with respect to the correlation scale of $Y$ (i.e., $L / \lambda \rightarrow 0$, with finite $L$ ), thus resembling a purely random, constant $K$ field, then $\lim _{L / \lambda \rightarrow 0} \sigma_{\varphi}^{2(2)}=0$ at any given location, $\xi$. Second, when the domain is very large with respect to the correlation scale of $Y$ (i.e., $L / \lambda \rightarrow \infty$; with finite $L$ ), then $\lim _{L / \lambda \rightarrow \infty} \sigma_{\varphi}^{2(2)}=0$ at any given location, $\xi$. Third, $\sigma_{\varphi}^{2(2)}$ has been found to attain a finite (non-zero) value in the proximity of the well $(\xi \rightarrow 0)$, by numerical integration of Eq. (8), meaning that the direction from which the particle is reaching the well is uncertain.

\section{Appendix B. Mean travel time}

Denoting $D_{1 q r}^{\prime}(r)=\left.\frac{\partial q_{r}^{\prime}(r, \varphi)}{\partial \varphi}\right|_{\varphi=\bar{\varphi}}$, multiplying Eq. (6) by $D_{1 q r}^{\prime}(r)$ and taking expectation yields:

$$
r \frac{\mathrm{d}\left\langle\varphi D_{1 q r}^{\prime}\left(r^{*}\right)\right\rangle}{\mathrm{d} r}=\left\langle\frac{q_{\theta}(r, \varphi)}{q_{r}(r, \varphi)} D_{1 q r}^{\prime}\left(r^{*}\right)\right\rangle
$$

Expanding $q_{\theta}$ and $q_{r}$ around the mean trajectory leads to the following differential equation for the $O\left(\sigma_{Y}^{2}\right)$ approximation of the cross covariance $C_{\varphi D_{1 q r}}^{(2)}\left(r, r^{*}, \mathbf{r}_{0}\right)=\left\langle\varphi^{\prime} D_{1 q r}^{\prime}\left(r^{*}\right)\right\rangle^{(2)}$ :

$$
\frac{\mathrm{d} C_{\varphi D_{1 q r}}^{(2)}\left(r, r^{*}, \mathbf{r}_{0}\right)}{\mathrm{d} r}=-\frac{2 \pi}{Q}\left\langle D_{1 q r}^{\prime}\left(r^{*}\right) q_{\theta^{\prime}}(r, \bar{\varphi})\right\rangle^{(2)}
$$

with the boundary condition $C_{\varphi D_{1 q r}}^{(2)}\left(r, r^{*}, \mathbf{r}_{0}\right)=C_{\varphi D_{1 q r}}^{0}$ (at $\left.r \equiv r_{0}\right)$. We note that $C_{\varphi D_{1 q r}}^{0}=0$ if the vector location $\mathbf{r}_{0}$ is deterministically known (i.e., $\theta_{0}^{\prime}=0$ ). Integrating Eq. (B2) with deterministic boundary condition yields:

$$
C_{\varphi D_{1 q r}}^{(2)}\left(r, r^{*}, \mathrm{r}_{0}\right)=-\left.\frac{2 \pi}{Q} \int_{r_{0}}^{r} \frac{\partial C_{q r q \theta}^{(2)}\left(r^{*}, \varphi^{*}, r_{\mathrm{II}}, \bar{\varphi}_{\mathrm{II}}\right)}{\partial \varphi^{*}}\right|_{\varphi^{*}=\bar{\varphi}^{*}} \mathrm{~d} r_{\mathrm{II}} .
$$



(B3):

The cross-moment $\left\langle\left.\varphi^{\prime} \frac{\partial q_{r}^{\prime}(r, \varphi)}{\partial \varphi}\right|_{\varphi=\bar{\varphi}}\right\rangle^{(2)}$ at zero lag is obtained by taking the limit $r^{*} \rightarrow r$ in Eq.

$$
C_{\varphi D_{1 q r}}^{(2)}\left(r, r^{*} \equiv r, \mathbf{r}_{0}\right)=-\left.\frac{2 \pi}{Q} \int_{r_{0}}^{r} \frac{\partial C_{q r q \theta}^{(2)}\left(r, \varphi, r_{\mathrm{II}}, \bar{\varphi}_{\mathrm{II}}\right)}{\partial \varphi}\right|_{\varphi=\bar{\varphi}} \mathrm{d} r_{\mathrm{II}} .
$$

Finally, substituting the above results into Eq. (11) allows obtaining the second order component of the mean travel time (Eq. (13)). Using the expressions for $C_{q r q \theta}{ }^{(2)}$ and of $\sigma_{q r}^{2(2)}$ proposed by Riva et al. (2001) leads to

$$
\left\langle t^{(2)}\left(\xi_{0}\right)\right\rangle=n \frac{\pi L^{2}}{2 Q} \sigma_{Y}^{2} \frac{L^{2}}{\lambda^{2}}\left\{\mathfrak{I}_{1}+\frac{\mathfrak{I}_{2}}{2 \pi}\right\}
$$

where

$$
\begin{aligned}
& \mathfrak{I}_{1}=-\int_{0}^{\xi_{0}} \int_{0}^{2 \pi} \int_{0}^{1} \xi\left\{\mathfrak{R}\left(\xi_{\mathrm{II}}, \theta_{\mathrm{II}}, \xi_{0}, \xi\right) \mathrm{dMC}\left(\xi_{\mathrm{II}}, \xi, \theta_{\mathrm{II}}\right)+2 \xi \mathrm{MG}\left(\xi, \xi_{\mathrm{II}}, \theta_{\mathrm{II}}\right) \operatorname{MC}\left(\xi_{\mathrm{II}}, \xi, \theta_{\mathrm{II}}\right)\right\} \\
& \times \mathrm{d} \xi_{\mathrm{II}} \mathrm{d} \theta_{\mathrm{II}} \mathrm{d} \xi \\
& \mathfrak{I}_{2}=\int_{0}^{\xi_{0}} \int_{0}^{2 \pi} \int_{0}^{2 \pi} \int_{0}^{1} \int_{0}^{1} \xi^{2} \operatorname{MG}\left(\xi, \xi_{\mathrm{I}}, \theta_{\mathrm{I}}\right)\left[\mathfrak{R}\left(\xi_{\mathrm{II}}, \theta_{\mathrm{II}}, \xi_{0}, \xi\right) \operatorname{dmc}\left(\xi_{\mathrm{I}}, \xi_{\mathrm{II}}, \vartheta\right)\right. \\
& \left.+\xi \operatorname{MG}\left(\xi, \xi_{\text {II }}, \theta_{\text {II }}\right) \operatorname{mc}\left(\xi_{\mathrm{I}}, \xi_{\text {II }}, \vartheta\right)\right] \mathrm{d} \xi_{\text {II }} \mathrm{d} \xi_{\mathrm{I}} \mathrm{d} \theta_{\mathrm{I}} \mathrm{d} \theta_{\mathrm{II}} \mathrm{d} \xi \quad \vartheta=\theta_{\mathrm{I}}-\theta_{\mathrm{II}} \\
& \mathfrak{R}\left(\xi_{i}, \theta_{i}, \xi_{0}, \xi\right)=\arctan \left[\frac{\xi \xi_{i}-\cos \theta_{i}}{\sin \theta_{i}}\right]-\arctan \left[\frac{\xi_{0} \xi_{i}-\cos \theta_{i}}{\sin \theta_{i}}\right]-\arctan \left[\frac{\xi-\xi_{i} \cos \theta_{i}}{\xi_{i} \sin \theta_{i}}\right] \\
& +\arctan \left[\frac{\xi_{0}-\xi_{i} \cos \theta_{i}}{\xi_{i} \sin \theta_{i}}\right] \quad i=\mathrm{I}, \mathrm{II} \\
& \operatorname{mc}\left(\xi_{\mathrm{I}}, \xi_{\mathrm{II}}, \vartheta\right)=\left[\cos \vartheta+2 \omega^{2}\left(\xi_{\mathrm{I}}-\xi_{\mathrm{II}} \cos \vartheta\right)\left(\xi_{\mathrm{II}}-\xi_{\mathrm{I}} \cos \vartheta\right)\right] \exp \left[-\omega^{2} d^{2}\right] \\
& \operatorname{MC}\left(\xi_{\mathrm{II}}, \xi, \theta_{\mathrm{II}}\right)=\left(\xi_{\mathrm{II}}-\xi \cos \theta_{\mathrm{II}}\right) \exp \left\lfloor-\omega^{2}\left(\xi^{2}+\xi_{\mathrm{II}}^{2}-2 \xi \xi_{\mathrm{II}} \cos \theta_{\mathrm{II}}\right)\right\rfloor \\
& \operatorname{dmc}\left(\xi_{\mathrm{I}}, \xi_{\mathrm{II}}, \vartheta\right)=\exp \left[-\omega^{2} d^{2}\right] \sin \vartheta\left\{2 \omega^{2}\left(\xi_{\mathrm{I}}^{2}+\xi_{\mathrm{II}}^{2}\right)-1+4 \omega^{4} \xi_{\mathrm{I}} \xi_{\mathrm{II}}\left[\cos \vartheta\left(\xi_{\mathrm{I}}^{2}+\xi_{\mathrm{II}}^{2}-\frac{3}{2 \omega^{2}}\right)\right.\right. \\
& \left.\left.-\xi_{I} \xi_{I I}\left(1+\cos ^{2} \vartheta\right)\right]\right\}
\end{aligned}
$$

$$
\operatorname{dMC}\left(\xi_{\mathrm{II}}, \xi, \theta_{\mathrm{II}}\right)=-\xi \sin \theta_{\mathrm{II}}\left(1-2 \omega^{2} \xi_{\mathrm{II}}^{2}+2 \omega^{2} \xi \xi_{\mathrm{II}} \cos \theta_{\mathrm{II}}\right) \exp \left[-\omega^{2}\left(\xi^{2}+\xi_{\mathrm{II}}^{2}-2 \xi \xi_{\mathrm{II}} \cos \theta_{\mathrm{II}}\right)\right]
$$

$$
\operatorname{MG}\left(\xi, \xi_{i}, \theta_{i}\right)=\frac{\xi \xi_{i}^{2}-\xi_{i} \cos \theta_{i}}{1+\xi^{2} \xi_{i}^{2}-2 \xi \xi_{i} \cos \theta_{i}}-\frac{\xi-\xi_{i} \cos \theta_{i}}{\xi^{2}+\xi_{i}^{2}-2 \xi \xi_{i} \cos \theta_{i}} \quad i=\text { I, II. }
$$




\section{Appendix C. Travel time variance}

The expressions of the different terms appearing in Eq. (16) are as follows:

$$
\begin{aligned}
& \mathfrak{I}_{3}=\int_{0}^{2 \pi} \int_{0}^{2 \pi} \int_{0}^{1} \int_{0}^{1}\left\{\cos \vartheta+2 \omega^{2}\left[\xi_{\mathrm{I}}-\xi_{\mathrm{II}} \cos \vartheta\right]\left[\xi_{\mathrm{II}}-\xi_{\mathrm{I}} \cos \vartheta\right]\right\} \exp \left[-\omega^{2} d^{2}\right] \\
& \times \tilde{P}\left(\xi_{0}, \xi_{\mathrm{I}}, \theta_{\mathrm{I}}\right) \tilde{P}\left(\xi_{0}, \xi_{\mathrm{II}}, \theta_{\mathrm{II}}\right) \mathrm{d} \xi_{\mathrm{I}} \mathrm{d} \xi_{\mathrm{II}} \mathrm{d} \theta_{\mathrm{I}} \mathrm{d} \theta_{\mathrm{II}} \quad \vartheta=\theta_{\mathrm{I}}-\theta_{\mathrm{II}}
\end{aligned}
$$

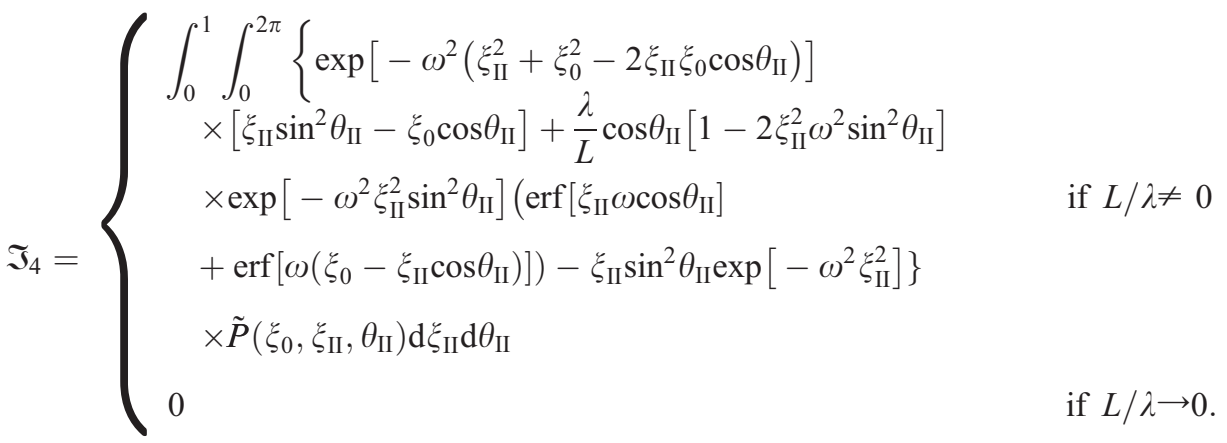

$$
\begin{aligned}
\tilde{P}\left(\xi_{0}, \xi_{i}, \theta_{i}\right)= & \frac{1-\xi_{i}^{2}}{\xi_{i}} \xi_{0} \cos \theta_{i}+\sin \left(2 \theta_{i}\right)\left\{\arctan \left(\cot \theta_{i}\right)\left(\xi_{i}^{2}-\frac{1}{\xi_{i}^{2}}\right)\right. \\
& \left.+\xi_{i}^{2} \arctan \left[\frac{\xi_{0}}{\xi_{i} \sin \theta_{i}}-\cot \theta_{i}\right]-\frac{1}{\xi_{i}^{2}} \arctan \left[\frac{\xi_{0} \xi_{i}}{\sin \theta_{i}}-\cot \theta_{i}\right]\right\} \\
& +\frac{\cos \left(2 \theta_{i}\right)}{2}\left[\xi_{i}^{2} \ln \left(\frac{\xi_{i}^{2}}{\xi_{i}^{2}+\xi_{0}^{2}-2 \xi_{i} \xi_{0} \cos \theta_{i}}\right)+\frac{1}{\xi_{i}^{2}} \ln \left[1+\xi_{i}^{2} \xi_{0}^{2}-2 \xi_{i} \xi_{0} \cos \theta_{i}\right]\right]
\end{aligned}
$$

$$
i=\mathrm{I}, \mathrm{II}
$$

\section{References}

Ballio, F., Guadagnini, A., 2004. Convergence assessment of numerical Monte Carlo simulations in groundwater hydrology. Water Resour. Res. 40, W04603. doi:10.1029/2003WR002876.

Cvetkovic, V., Cheng, H., Wen, X.-H., 1996. Analysis of nonlinear effects on tracer migration in heterogeneous aquifers using Lagrangian travel time statistics. Water Resour. Res. 32 (6), 1671-1680.

Cvetkovic, V., Dagan, G., Cheng, H., 1998. Contaminant transport in aquifers with spatially variable hydraulic and sorption parameters. Royal Society of London, vol. 454, pp. 2173-2207.

Dagan, G., 1984. Solute transport in heterogeneous porous formations. J. Fluid Mech. 145, 151-177.

Dagan, G., Indelman, P., 1999. Reactive solute transport in flow between a recharging and a pumping well in a heterogeneous aquifer. Water Resour. Res. 35 (12), 3639-3647.

Dagan, G., Cvetkovic, V., Shapiro, A.M., 1992. A solute flux approach to transport in heterogeneous formations: 1. The general framework. Water Resour. Res. 28 (5), 1369-1376.

Feyen, L., Beven, K.J., De Smedt, F., Freer, J., 2001. Stochastic capture zone delineation within the generalized likelihood uncertainty estimation methodology: conditioning on head observations. Water Resour. Res. 37 (3), $625-638$.

Franzetti, S., Guadagnini, A., 1996. Probabilistic estimation of well catchments in heterogeneous aquifers. J. Hydrol. 174 (1-2), 149-171. 
Gómez-Hernández, J.J., 1991. A stochastic approach to the simulation of block conductivity fields conditioned upon data measured at a smaller scale. PhD Dissertation, Stanford Univ.

Guadagnini, A., Franzetti, S., 1999. Time-related capture zones for contaminants in randomly heterogeneous formations. Ground Water 37 (2), 253-260.

Guadagnini, A., Sánchez-Vila, X., Riva, M., De Simoni, M., 2003. Mean travel time of conservative solutes in randomly heterogeneous unbounded domains under mean uniform flow. Water Resour. Res. 39 (3), 1050. doi:10.1029/ 2002WR001811.

Indelman, P., Dagan, G., 1999. Solute transport in divergent radial flow through heterogeneous porous media. J. Fluid Mech. 384, 159-182.

Lawrence, A.E., Sánchez-Vila, X., Rubin, Y., 2002. Conditional moments of the breakthrough curves of kinetically sorbing solute in heterogeneous porous media using multirate mass transfer models for sorption and desorption. Water Resour. Res. 38 (11), 1248. doi:10.1029/2001WR001006.

Lessoff, S.C., Indelman, P., 2004. Stochastic determination of three-dimensional capture zones for a fully penetrating well. Water Resour. Res. 40 (3), W03508. doi:10.1029/2003WR002703.

Rajaram, H., 1997. Time and scale dependent effective retardation factors in heterogeneous aquifers. Adv. Water Resour. 20 (4), 217-230.

Riva, M., Guadagnini, A., Ballio, F., 1999. Time related capture zones for radial flow in two-dimensional randomly heterogeneous media. Stoch. Environ. Res. Risk Assess. 13 (3), 217-230.

Riva, M., Guadagnini, A., Neuman, S.P., Franzetti, S., 2001. Radial flow in a bounded randomly heterogeneous aquifer. Transp. Porous Media 45, 139-193.

Sánchez-Vila, X., Guadagnini, A., 2005. Travel time and trajectory moments of conservative solutes in three dimensional heterogeneous porous media under mean uniform flow. Adv. Water Resour. 28 (5), 429-439.

Sánchez-Vila, X., Rubin, Y., 2003. Travel time moments for sorbing solutes in heterogeneous domains under nonuniform flow conditions. Water Resour. Res. 39 (4), 1086. doi:10.1029/2002WR001399.

Sánchez-Vila, X., Meier, P.M., Carrera, J., 1999. Pumping tests in heterogeneous aquifers: an analytical study of what can be obtained from their interpretation using Jacob's method. Water Resour. Res. 35 (4), 943-952.

Shapiro, A.M., Cvetkovic, V.D., 1988. Stochastic analysis of solute arrival time in heterogeneous porous media. Water Resour. Res. 24 (10), $1711-1718$.

Thiem, G., 1906. Hydrologische Methoden. J.M. Gebhardt, Leipzig.

van Leeuwen, M., Te Stroet, C.B.M., Butler, A.P., Tompkins, J.A., 2000. Stochastic determination of well capture zones conditioned on regular grids of transmissivity measurements. Water Resour. Res. 36 (4), 949-957.

Varljen, M.D., Shafer, J.M., 1991. Assessment of uncertainty in time-related capture zones using conditional simulation of hydraulic conductivity. Ground Water 29 (5), 737-748.

Zhang, D., Andricevic, R., Sun, A.Y., Hu, X., He, G., 2000. Solute flux approach to transport through spatially nonstationary flow in porous media. Water Resour. Res. 36 (8), 2107-2121. 